\title{
On the dissipation and dispersion of entropy waves in heat transferring channel flows
}

A. Fattahi, S. M. Hosseinalipour, and N. Karimi

Citation: Physics of Fluids 29, 087104 (2017); doi: 10.1063/1.4999046

View online: http://dx.doi.org/10.1063/1.4999046

View Table of Contents: http://aip.scitation.org/toc/phf/29/8

Published by the American Institute of Physics

\section{Articles you may be interested in}

Why do we live for much less than 100 years? A fluid mechanics view and approach

Physics of Fluids 29, 081903 (2017); 10.1063/1.4998717

Turbulent boundary layer over a divergent convergent superhydrophobic surface

Physics of Fluids 29, 085112 (2017); 10.1063/1.4999938

Turbulent kinetic energy budget in the boundary layer developing over an urban-like rough wall using PIV Physics of Fluids 29, 085113 (2017); 10.1063/1.4997205

Morphology of drop impact on a superhydrophobic surface with macro-structures

Physics of Fluids 29, 082104 (2017); 10.1063/1.4997266

Instability in a channel with grooves parallel to the flow

Physics of Fluids 29, 084104 (2017); 10.1063/1.4997950

Identification of coherent structures in the flow past a NACA0012 airfoil via proper orthogonal decomposition Physics of Fluids 29, 085104 (2017); 10.1063/1.4997202

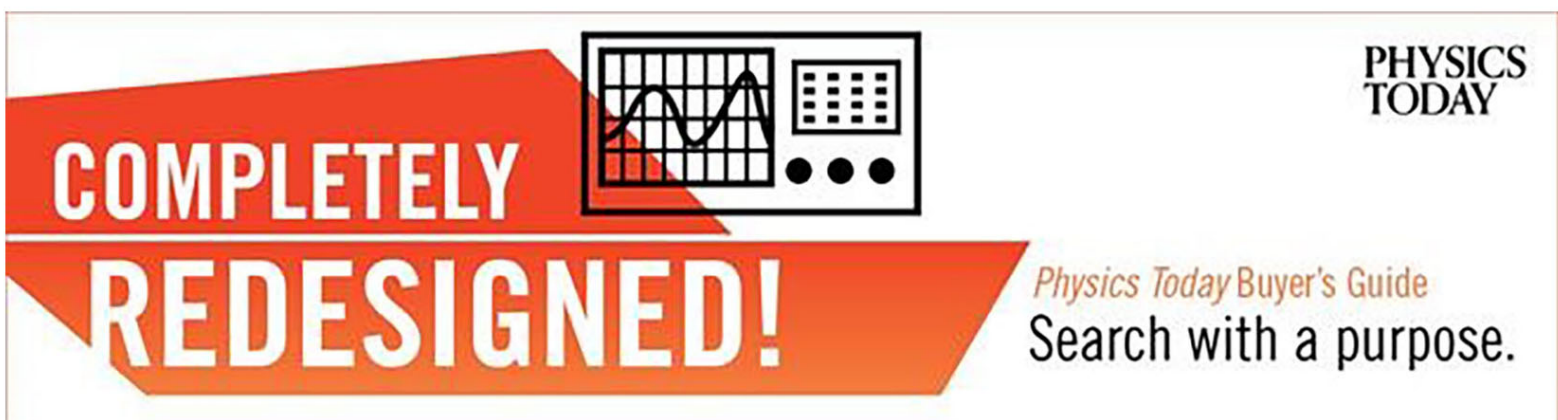




\title{
On the dissipation and dispersion of entropy waves in heat transferring channel flows
}

\author{
A. Fattahi, ${ }^{1,2}$ S. M. Hosseinalipour, ${ }^{1, a)}$ and N. Karimi ${ }^{3}$ \\ ${ }^{1}$ School of Mechanical Engineering, Iran University of Science and Technology, Tehran, Iran \\ ${ }^{2}$ Department of Mechanical Engineering, University of Kashan, Kashan, Iran \\ ${ }^{3}$ School of Engineering, University of Glasgow, Glasgow G12 8QQ, United Kingdom
}

(Received 16 March 2017; accepted 4 August 2017; published online 28 August 2017)

\begin{abstract}
This paper investigates the hydrodynamic and heat transfer effects on the dissipation and dispersion of entropy waves in non-reactive flows. These waves, as advected density inhomogeneities downstream of unsteady flames, may decay partially or totally before reaching the exit nozzle, where they are converted into sound. Attenuation of entropy waves dominates the significance of the subsequent acoustic noise generation. Yet, the extent of this decay process is currently a matter of contention and the pertinent mechanisms are still largely unexplored. To resolve this issue, a numerical study is carried out by compressible large eddy simulation of the wave advection in a channel subject to convective and adiabatic thermal boundary conditions. The dispersion, dissipation, and spatial correlation of the wave are evaluated by post-processing of the numerical results. This includes application of the classical coherence function as well as development of nonlinear quantitative measures of wave dissipation and dispersion. The analyses reveal that the high frequency components of the entropy wave are always strongly damped. The survival of the low frequency components heavily depends on the turbulence intensity and thermal boundary conditions of the channel. In general, high turbulence intensities and particularly heat transfer intensify the decay and destruction of the spatial coherence of entropy waves. In some cases, they can even result in the complete annihilation of the wave. The current work can therefore resolve the controversies arising over the previous studies of entropy waves with different thermal boundary conditions. Published by AIP Publishing. [http://dx.doi.org/10.1063/1.4999046]
\end{abstract}

\section{INTRODUCTION}

Over forty years ago, it was shown theoretically that advection of a density inhomogeneity through a region of mean flow acceleration generates sound. ${ }^{1,2}$ The problem was then termed entropy noise and correspondingly the density inhomogeneities or hot spots were called entropy waves. ${ }^{3,4}$ It has been demonstrated that unsteady flames can generate entropy waves, which are advected downstream by the mean flow and may reach the exit nozzle of the combustor., ${ }^{3,5,6}$ Due to the significant acceleration of the mean flow in the exit nozzle and the early stages of the high pressure turbine, entropy waves can be converted into acoustic waves. ${ }^{7,8}$ This forms a source of noise in the gas turbine, which originates from the combustion process. However, the existence of this noise is subject to the passage of entropy waves through the downstream nozzles. Hence, entropy noise is regarded as indirect combustion noise to also be differentiated from the direct combustion noise generated primarily by turbulence. ${ }^{3,9}$ In recent years, the growing importance of clean and quiet combustion in land-based gas turbines and aero-engines has renewed the interest in entropy noise. ${ }^{10,11}$

The process of conversion of entropy waves into acoustic waves was first modeled by Marble and Candel in their seminal analytical work. ${ }^{12}$ This one-dimensional, compact, linear theory uses compressible Euler equations and develops acoustic

\footnotetext{
${ }^{a)}$ Author to whom correspondence should be addressed: alipour@iust.ac.ir
}

reflection and transmission coefficients for an incident entropy wave in subcritical and supercritical nozzles. ${ }^{12}$ For more than three decades, the formulations of Marble and Candel ${ }^{12}$ were the main tools for modeling the acoustic effects of entropy waves in combustion dynamics. Over the last ten years, a number of attempts were made to release the assumptions made by Marble and Candel ${ }^{12}$ about the linearity of the system and compactness of the nozzle. ${ }^{13-18}$ These resulted in improving the predictability of the acoustic conversion of entropy waves. For instance, the results of well-controlled experiments of Bake et $a l .{ }^{19,20}$ in an entropy wave generator were initially in fair agreement with the classical prediction of Marble and Candel. ${ }^{12}$ The causes of disagreement were explained and mostly resolved in the recent refinements of the original theory. ${ }^{11,15}$ Despite these theoretical developments, most acoustic models still assume entropy waves to be one-dimensional and preserved during advection in the upstream channel, while the validity of these assumptions is unclear.

There is now a body of experimental ${ }^{21,22}$ and theoreti$\mathrm{cal}^{11,23,24}$ studies showing that entropy noise could influence the thermoacoustic instability of laboratory scale combustors. Yet, the studies conducted in real engines revealed very few effects. ${ }^{25,26}$ This was such that some authors considered entropy waves to be of little significance to thermoacoustics of gas turbines. ${ }^{27}$ These two opposing viewpoints have set an ongoing debate on the problem of entropy noise. Given that the conversion of entropy waves into sound is well demonstrated, the central contention is on the survival of entropy waves 
in the combustor. In a semi-analytical work, Sattelmayer ${ }^{27}$ examined advection of entropy waves in a simple gas turbine combustor represented by a non-reactive, adiabatic duct flow. The results were subsequently used in a theoretical analysis to show that a scalar disturbance (e.g., entropy wave) would be totally dispersed in the combustor. ${ }^{27}$ This conclusion was rebutted by Morgans et al. ${ }^{28}$ through conduction of a direct numerical simulation of the advection of entropy waves by an adiabatic, incompressible channel flow. Morgans et al. ${ }^{28}$ modeled entropy waves as passive scalars and argued that they remain mostly conserved. They further showed that the dispersion of these waves in their investigated configuration is negligibly small. ${ }^{28}$

Limited two-dimensional data obtained from thermoacoustically unstable combustors indicate that entropy waves may become spatially uncorrelated and violate the assumption of one-dimensionality. ${ }^{29}$ Nevertheless, due to the flow complexity in such configurations, making any precise conclusion about the evolution of entropy waves is still very difficult. ${ }^{30}$ Further, most recent studies have shown that entropy waves do not necessarily propagate as passive scalars and can affect the base flow. ${ }^{31}$ It is, therefore, unclear if the conventional one-dimensional, passive scalar treatment is always representative of the real entropy waves. ${ }^{32,33}$ Besides, currently there is limited understanding of the decay of amplitude or dissipation of entropy waves as they travel from the reactive region to the exit nozzle of the combustor. It is also unknown if the wave dissipation is uniform across all wavelengths or there exists a selective process in which some wavelengths are dissipated more strongly. Such a process would have direct consequences on the frequency content of the resultant entropy noise. Furthermore, little is known about the influence of the flow specifications on the wave dispersion.

Real gas turbine combustors include complex aerodynamics, highly turbulent flow, and heat transfer to the surrounding environment. The studies of Morgans et al. ${ }^{28}$ and Sattelmayer ${ }^{27}$ considered turbulent flows and assumed adiabatic combustors with simple aerodynamics. Although disagreeing on the extent, they both demonstrated that hydrodynamic non-uniformities can destroy the entropy wave. A heat transferring flow further includes thermal non-uniformities, which could feature analogous decaying effects on the entropy wave. Nonetheless, so far the effects of thermal non-uniformities of the base flow on the attenuation of entropy waves have been ignored. To explore these, the current work releases the assumption of adiabatic advective flows. The heat transfer and turbulence effects are investigated by simulating advection of entropy waves in a convectively cooled channel containing a compressible fluid flow. The advection process is also simulated in an adiabatic channel for two different values of turbulence intensity. The temporal and spatial evolutions of the wave are then analyzed by employing linear and non-linear postprocessing techniques. It will be shown that heat transfer and inlet turbulence intensity can significantly increase the attenuation of the entropy wave and destroy its spatial correlation. The current work is exclusively concerned with gaseous flows that correspond to combustion products of lean premixed flames. In other types of flames, the combustion products may include a secondary phase in the form of particulates. ${ }^{34}$ This can potentially modify the physical processes discussed in this work.

\section{NUMERICAL AND THEORETICAL METHODS}

\section{A. Problem configuration and boundary conditions}

The computational domain includes a fully developed turbulent flow between two parallel plates, similar to that investigated in Ref. 28. Figure 1 shows a schematic view of the configuration under investigation, in which the length, height, and width are, respectively, $24 \pi d, 2 d$, and $\pi d$ and $d=2.5 \mathrm{~mm}$. Figure 1 further shows that the external surfaces of the channel may include convective heat transfer to the surroundings through the upper and lower walls. The convective heat flux on the external surface of the wall $\left(q^{\prime \prime}\right)$ is calculated by

$$
q^{\prime \prime}=h\left(T_{\text {wall }}-T_{\infty}\right),
$$

in which $T_{\text {wall }}$ and $T_{\infty}$ are, respectively, the temperatures of the duct wall and external cooling flow and $h$ is the heat convection coefficient on the external surfaces of the duct. Also, specified in Fig. 1 are four cross sections $(A, B, C$, and $D)$ along the channel and three points $(1,2$, and 3$)$ situated transversally across the channel. The working fluid is air, and the flow

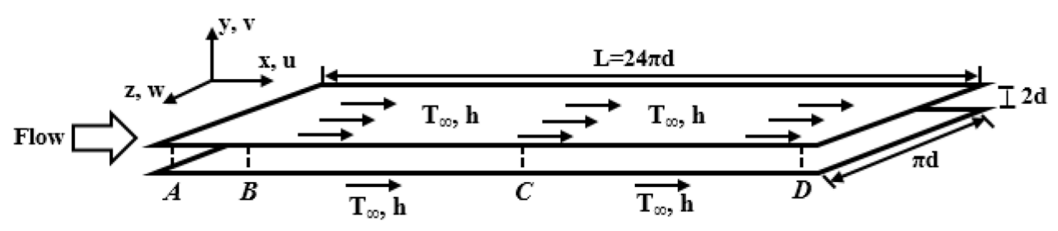

(a)

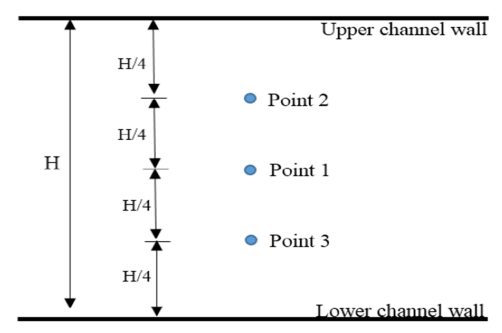

(b)
FIG. 1. (a) Schematic configuration of the channel and the longitudinal position of cross sections, $A$ at $x / L$ $=0.005, B$ at $x / L=0.125, C$ at $x / L=0.5$, and $D$ at $x / L$ $=0.995$, (b) cross section of the channel with points $1-3$ in the transversal direction. 
temperature and pressure at the inlet are maintained constant at $300 \mathrm{~K}$ and $1 \mathrm{bar}$, respectively. Further, the values of heat transfer coefficient and ambient temperature are, respectively, $100 \mathrm{~W} / \mathrm{m}^{2} \mathrm{~K}$ and $273 \mathrm{~K}^{34}$ In the proceeding analyses, the turbulent Reynolds number is defined as

$$
\operatorname{Re}_{\tau}=u_{\tau} h / v,
$$

and similar to that in the work of Morgans et al. ${ }^{28}$ its value is assumed to be 180 . In Eq. (2), $h$ is the channel half-height, $v$ is the kinematic viscosity, and $u_{\tau}$ is the friction velocity. The latter is defined as follows:

$$
u_{\tau}=\sqrt{\frac{\tau_{w}}{\rho}}
$$

in which $\tau_{w}$ is the flow shear stress on the wall. The Reynolds number on the basis of hydraulic diameter and bulk velocity is set to 13000 . Further, a fully developed velocity profile is applied at the inlet and periodic boundary conditions in the span-wise and streamwise directions and also no-slip conditions are set on the walls. Zero axial gradients for all outlet flow variables are imposed and adiabatic or convective thermal boundary conditions are applied on the channel walls.

Decomposition of the thermodynamic properties into steady and perturbation components and a subsequent linearization of Gibbs and ideal gas relations result in the following equations: ${ }^{10,32}$

$$
\begin{gathered}
\frac{s^{\prime}}{c_{p}}=\frac{T^{\prime}}{\bar{T}}-\left(\frac{\gamma-1}{\gamma}\right) \frac{p^{\prime}}{\bar{p}}, \\
\frac{P^{\prime}}{P}=\frac{\rho^{\prime}}{\rho}+\frac{T^{\prime}}{T} .
\end{gathered}
$$

In the absence of acoustic fluctuations (i.e., $\mathrm{p}^{\prime}$ being negligible), it may be concluded that $\frac{\mathrm{s}^{\prime}}{\mathrm{c}_{\mathrm{p}}} \cong \frac{\mathrm{T}^{\prime}}{\overline{\mathrm{T}}}$ and also $\frac{\rho^{\prime}}{\rho} \cong \frac{\mathrm{T}^{\prime}}{\mathrm{T}},{ }^{11,28}$ which indicates that the temperature (density) and entropy disturbances scale similarly. This similarity is the rationale behind generating the entropy wave by a transient temperature function at the channel inlet. The same argument can be made through resorting to the nonlinear versions of Eq. (4). As an essential point, it is stressed that the current study does not include any linearization of the governing equations. Equation (4) also shows that in the absence of acoustic waves and by excluding broadband fluctuations of pressure, entropy waves reduce to temperature or density waves. ${ }^{10} \mathrm{~A}$ temperature pulse is added to the air flow using a linear increment and an exponential decrement function. ${ }^{20}$ Unlike the induced heat source function of Morgans et al.,${ }^{28}$ this has the capacity of practical implementation $^{20}$ and a lower potential for inducing numerical errors. This high temperature region, with the amplitude of 1.1 times the absolute temperature of the base flow, then advects through the channel and develops an entropy wave.

\section{B. Mathematical model and numerical schemes}

Decay of entropy waves is primarily due to turbulent mixing, which is dominated by eddy dynamics in the flow. As a result, accurate simulation of eddies is an essential necessity for the investigation of decaying entropy waves. The conventional Reynolds average Navier-Stokes (RANS) equations cannot capture eddies, while a direct numerical simulation (DNS) can be prohibitively expensive for the cases studied in the current problem. Hence, as an optimal solution, large eddy simulation (LES) is employed in the present work.

In LES, the governing equations are obtained through filtering the Navier-Stokes equations on the physical domain. Filtered equations can, then, separate large- and small-scale eddies in the flow. The filtration process leads to the addition of extra terms to the momentum and energy equations. These are referred to as subgrid-scale (SGS) stresses and enthalpy flux that are unknown and hence demand modeling. In general, the flow property $\psi$ is decomposed into large-scale $\bar{\psi}$ and small-scale $\psi^{\prime}$, where $\bar{\psi}$ is expressed as

$$
\bar{\psi}(x)=\int_{V} \psi\left(x^{\prime}\right) G\left(x, x^{\prime}\right) d x^{\prime}, x^{\prime} \epsilon V .
$$

In Eq. (5), $\psi$ is the original (unfiltered) function, $x^{\prime}$ is the spatial coordinate, $x$ is the spatial coordinate after filtering, $G$ is the filter function, and $V$ is the volume of a computational cell. ${ }^{35}$ For a compressible flow of a Newtonian fluid, the filtered mass and momentum equations are expressed by

$$
\begin{gathered}
\frac{\partial \bar{\rho}}{\partial t}+\frac{\partial}{\partial x_{j}}\left(\bar{\rho} \tilde{u}_{j}\right)=0 \\
\frac{\partial}{\partial t}\left(\bar{\rho} \tilde{u}_{i}\right)+\frac{\partial}{\partial x_{j}}\left(\bar{\rho} \tilde{u}_{i} \tilde{u}_{j}\right)=-\frac{\partial \bar{P}}{\partial x_{i}}+\frac{\partial}{\partial x_{j}}\left(\bar{\tau}_{i j}-\bar{\rho}\left(\widetilde{u_{i} u_{j}}-\tilde{u}_{i} \tilde{u}_{j}\right)\right)
\end{gathered}
$$

In these equations, $\rho, u$, and $p$ are fluid density, velocity, and pressure, respectively. The overbar symbol denotes the spatial filtering used in LES and the symbol $\sim$ denotes the Favre filtering, defined as $\tilde{\psi}=\overline{\rho \psi} / \bar{\rho}$ for an arbitrary variable $\psi \cdot{ }^{36}$ Further, $\tau_{i j}$ is the subgrid-scale stress tensor given by

$$
\tau_{i j}=\rho \overline{u_{i} u_{j}}-\rho \bar{u}_{i} \bar{u}_{j}
$$

Turbulent structures belonging to the inertial range turbulent spectrum are directly simulated, using a suitably dense grid, while the smaller grid scales of motion (subgrid-scale or SGS) are parameterized with a specific model. ${ }^{36}$ The subgrid-scale turbulent stresses are computed as follows:

$$
\tau_{i j}^{S G S}-\frac{1}{3} \delta_{i j} \tau_{k k}^{S G S}=-2 \mu_{S G S}\left(\tilde{S}_{i j}-\frac{1}{3} \delta_{i j} \tilde{S}_{k k}\right),
$$

where $\mu_{S G S}$ is the subgrid-scale viscosity and $\tilde{S}_{i j}$ is the strain rate tensor for the resolved scales and is defined by

$$
\tilde{S}_{i j}=\frac{1}{2}\left(\frac{\partial \tilde{u}_{j}}{\partial x_{i}}+\frac{\partial \tilde{u}_{i}}{\partial x_{j}}\right) .
$$

$\tau_{k k}^{S G S}$ is the isentropic part of the subgrid-scale tensor, related to the subgrid-scale kinetic energy,

$$
\tau_{k k}^{S G S}=\bar{\rho}\left(\widetilde{u_{k} u_{k}}-\tilde{u}_{k} \tilde{u}_{k}\right)=2 \bar{\rho} k_{S G S} .
$$

Different models have been recommended in the literature for the calculation of the subgrid-scale turbulent viscosity $\left(\mu_{\mathrm{t}}\right){ }^{36}$ Here, the Wall-Adapting Local Eddy-Viscosity (WALE) model ${ }^{35}$ was chosen. This is because of the 
advantages of this model for the wall bounded flows in comparison with the commonly used Smagorinsky-Lilly model. ${ }^{37}$ The WALE model considers zero turbulent viscosity for laminar shear flows in contrast with the Smagorinsky-Lilly model ${ }^{37}$ that assumes non-zero turbulent viscosity. This enhances the accuracy of the WALE model in the treatment of the laminar zones of the domain. It is also noted that this model has been employed in the recent LES studies of thermoacoustic instabilities. ${ }^{38,39}$ In the WALE model, $\mu_{t}$ is calculated from

$$
\mu_{t}=\rho L_{s}^{2} \frac{\left(S_{i j}^{d} S_{i j}^{d}\right)^{3 / 2}}{\left(\bar{S}_{i j} \bar{S}_{i j}\right)^{5 / 2}+\left(S_{i j}^{d} S_{i j}^{d}\right)^{5 / 4}},
$$

where $S_{i j}^{d}=\frac{1}{2}\left(\bar{g}_{i j}^{2}+\bar{g}_{j i}^{2}\right)-\frac{1}{3} \delta_{i j} \bar{g}_{k k}^{2}$ and $\bar{g}_{i j}=\frac{\partial \bar{u}_{i}}{\partial x_{j}} . L_{s}$ is the mixing length of the subgrid-scale and is computed by ${ }^{35}$

$$
L_{S}=\min \left(\kappa d, C_{w} V^{1 / 3}\right) .
$$

Further details of the $L_{S}$ function are provided by Nicoud and Ducros. ${ }^{35}$ In Eq. (13), $\kappa$ is von-Karman's constant, which is set to 0.4 in the current work, ${ }^{35}$ and $d$ is the distance to the closest wall for each computational cell. Filtration of the flow energy equation results in the following equation:

$$
\begin{aligned}
& \frac{\partial}{\partial t}\left(\bar{\rho} \tilde{h}_{s}\right)+\frac{\partial}{\partial x_{j}}\left(\bar{\rho} \tilde{u}_{j} \tilde{h}_{s}\right) \\
& \quad=\frac{D \bar{P}}{D t}+\bar{\tau}_{i j}: \frac{\partial \tilde{u}_{j}}{\partial x_{i}}+\frac{\partial}{\partial x_{j}}\left(\frac{\mu}{\operatorname{Pr}} \frac{\partial \tilde{h}_{s}}{\partial x_{j}}-\bar{\rho}\left(\widetilde{u_{j} h_{s}}-\tilde{u}_{j} \tilde{h}_{s}\right)\right),
\end{aligned}
$$

in which $\bar{h}_{s}$ and $\operatorname{Pr}_{S G S}$ are the sensible enthalpy and sub-grid scale Prandtl number, respectively. The term $\bar{\rho}\left(\widetilde{u_{j} h_{s}}-\tilde{u}_{j} \tilde{h}_{s}\right)$ is the subgrid enthalpy flux and is given by

$$
\bar{\rho}\left(\widetilde{u_{j} h_{s}}-\tilde{u}_{j} \tilde{h}_{s}\right)=-\frac{\mu_{S G S}}{\operatorname{Pr}_{S G S}} \frac{\partial \tilde{h}_{s}}{\partial x_{j}} .
$$

Finite volume on a Cartesian grid was employed to solve the unsteady, compressible Navier-Stokes equations with the coupled algorithm that solves the momentum and continuity equations together. The spatial discretization was performed using a second-order backward and bounded scheme. Time derivatives are discretized with a second-order backward difference scheme. The time step was set such that the Courant-Friedrichs-Lewy $(C F L)$ number was always lower than unity. ${ }^{40}$ Based on the definition of the $C F L$ number, a constant time step was set to $3.5 \mu \mathrm{s}$. This is

$$
\Delta t=C F L \Delta x_{\min } / u_{1 \max },
$$

where $\Delta x_{\text {min }}$ is the minimum grid width and $\mathrm{u}_{1 \max }$ is the maximum longitudinal velocity. In each time step, density of the compressible fluid flow was renewed via the ideal gas relation. Although the current simulation is compressible, the acoustic waves are not of interest and the investigated very low Mach number flow is essentially silent. For this reason, the bulk flow velocity is used in the definition of the $C F L$ number.

To achieve fast convergence of computations, simulations were initialized with a numerical solution obtained by a steady
RANS with a standard k- $\varepsilon$ model. Recording of the results started when all the initial transients were washed out and a semi-steady state operating condition was reached. Prior to recording of the results, a duration twice as the advection time of a massless particle elapsed to ensure the collection of reliable data. This was determined by comparing the variations of the root-mean-square of velocities versus $\mathrm{y}^{+}$at a few points for consecutive advections of the massless particle through the channel.

\section{Grid independency and validations}

To find out the optimal grid size, the ratio of the area under the temperature-time graph at the inlet to that at the outlet was first computed for a range of grid sizes. The results showed that a grid consisting of 490000 cells was sufficiently fine to ensure grid independency of the solution. A non-uniform grid in the $\mathrm{y}$ direction was implemented with maximum and minimum spatial resolutions of 0.36 and $0.020 \mathrm{~mm}$, respectively. The cells in the other directions have a uniform size of $0.30 \mathrm{~mm}$. The grid was further rechecked by examining the $\mathrm{LES}_{\mathrm{IQ}_{\mathrm{k}}}$ index $^{41}$ for various grid sizes. This is defined as follows: ${ }^{41}$

$$
L E S_{-} I Q_{k}=\frac{k^{r e s}}{k^{t o t}},
$$

which is an assessment index for the quality of a LES mesh size. Here, $k^{\text {res }}$ is the resolved kinetic energy and $k^{\text {tot }}$ is the total kinetic energy. The adequate values of $L E S_{-} I Q_{k}$ for high Reynolds number flows are more than $0.75 .{ }^{42}$ The minimum $L E S_{-} I Q_{k}$ in the current study was 0.91 , confirming the adequacy of the number of cells. The grid, however, should be finer near the walls. Hence, the dimensionless velocity $\left(u^{+}\right)$and wall distance $\left(y^{+}\right)$for turbulent flows were also considered. These are

$$
U^{+}=\frac{U}{u_{\tau}}, y^{+}=\frac{\rho u_{\tau} \Delta y}{\mu},
$$

where $\Delta y$ is the distance of the cell from the wall and $U$ is the time averaged flow velocity. Figure 2 shows the variation of the computed $U^{+}$against $y^{+}$, which agrees well with the

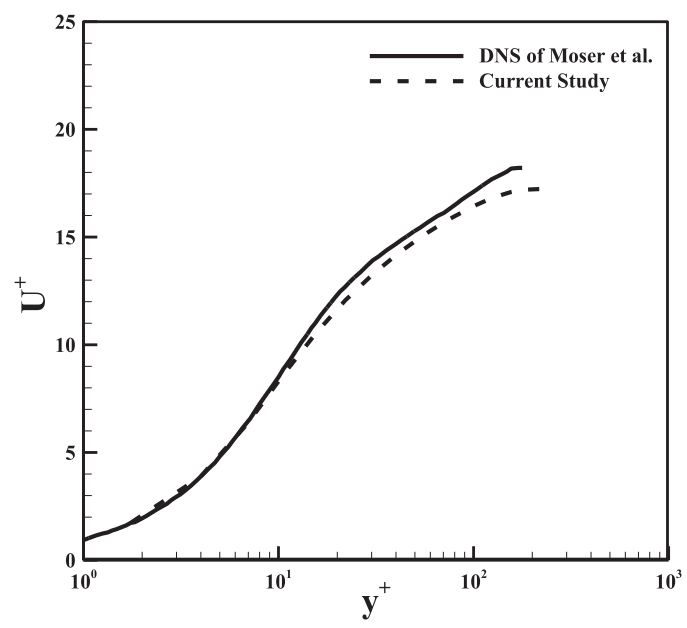

FIG. 2. Comparison between the current LES and DNS of Moser and Mansour. $^{43}$ 


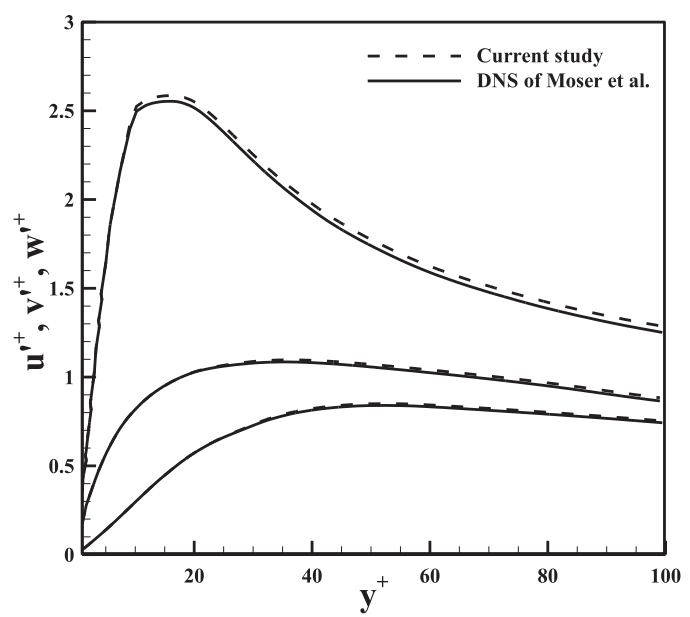

FIG. 3. Comparison between the current LES and DNS of Moser and Mansour; ${ }^{43} \mathrm{u}^{\prime+}$ has the highest and $\mathrm{w}^{\prime+}$ has the lowest amplitudes.

DNS results of Moser and Mansour. ${ }^{43}$ Figure 3 demonstrates a comparison between the DNS of Moser and Mansour ${ }^{43}$ and the current LES for the root-mean-square of the velocity components $\left(u^{\prime+}, v^{\prime+}, w^{\prime+}\right)$ versus wall distance $\left(y^{+}\right)$. A good agreement with a maximum discrepancy of less than $2 \%$ is evident in this figure. Moreover, in keeping with the LES literature, ${ }^{37} y^{+}$for the nearest cell to the wall is less than unity (about 0.9). Although the work of Moser and Mansour ${ }^{43}$ was on the basis of an incompressible flow, it had the same geometry and boundary conditions as the current work. It is also noted that the very low Mach number of the flow in the current study makes it comparable to an incompressible flow. Nonetheless, to establish further validation, the computed history of the temperature increment was compared against the experimental results of Bake et al. ${ }^{20}$ (see Fig. 4). Due to the unknown spanwise and altitudinal position of the thermocouple in Ref. 20, the mass-weighted average of the flow temperature is shown in Fig. 4. This is the reason for the existence of a small difference between the two sets of data at the wave rear. The near wall region was included in the mass-weighted average of the numerical work but not in the experimental data of

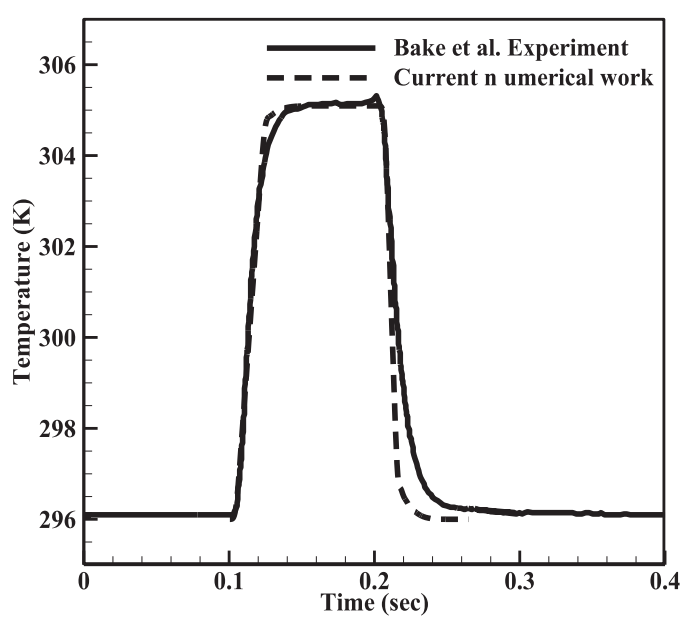

FIG. 4. Comparison of the computed temperature increment versus time, with the measurements of Bake et al. ${ }^{20}$

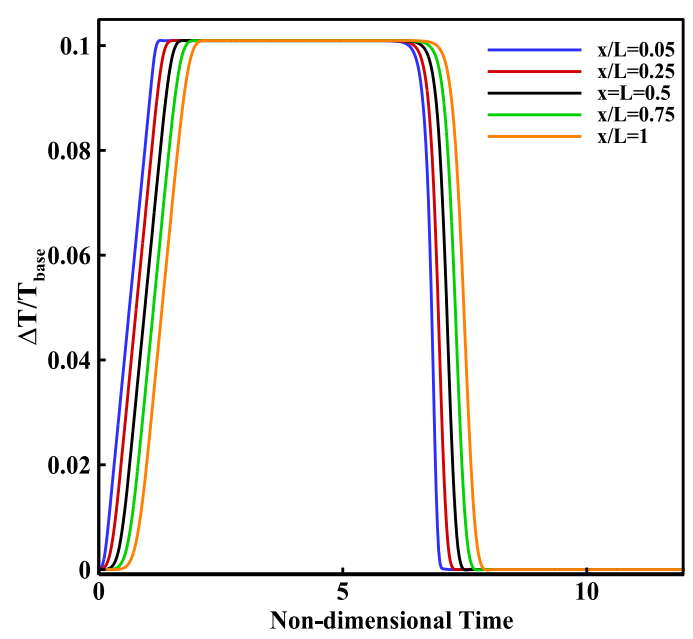

FIG. 5. The ratio of the increment to the base temperature value in various cross sections of an Eulerian flow.

Ref. 20. The smaller mean velocities near the walls of the channel lead to spreading at the rear part of the temperature pulse. ${ }^{28}$ Figure 4 shows that with the exception of some small discrepancies at the rear of the pulse, a favorable agreement exists between the computed data and the measured ones. The current numerical simulations were further compared with the DNS of Jongwoo et al. ${ }^{44}$ on mixed thermal convection, which resulted in an excellent agreement in the velocity profiles and mean Nusselt number.

Physical diffusion, as a major contributor to the dispersion and dissipation of entropy waves, is of primary importance in the current problem. Fluid conductivity and viscosity are the two physical diffusion agents involved in energy and momentum equations. Numerical diffusion, however, may act as a non-physical source that influences the results in an erroneous way. The implementation of second-order discretization and the use of fine grids in the current study reduce the numerical diffusion significantly. Nevertheless, the extent of non-physical diffusion was further examined in the framework of an Eulerian flow. An inviscid flow with zero thermal conductivity was considered. Similar to that in the main simulations, the inlet temperature of the flow was set to $300 \mathrm{~K}$ and entropy waves were induced at the inlet. The ratio of the mass-weighted average of the temperature increment to the base temperature in different cross sections along the channel is shown in Fig. 5. It is clear from this figure that the overall behavior of the wave remains unchanged. There is a minor difference in the area under the graph between the inlet and the outlet, and the maximum change of the wave width is less than $3 \%$. It is therefore concluded that the numerical diffusion remains negligible and the diffusion observed in the simulation is exclusively of physical nature.

\section{Coherence analysis}

Spectral coherence can examine the linear relation between two signals in the frequency domain. The coherence (sometimes called magnitude-squared coherence) between two signals $x(t)$ and $y(t)$ is a real-valued function, defined as ${ }^{45}$ 


$$
C_{x y}(f)=\frac{\left(G_{x y}(f)\right)^{2}}{G_{x x}(f) G_{y y}(f)},
$$

where $G_{x x}(f)$ and $G_{y y}(f)$ are the auto-spectral densities of $x$ and $y$, respectively. These functions are calculated using the mathematical expectation operator

$$
E[x(t)]=\int_{-\infty}^{+\infty} x(t) P(t) d t,
$$

where $P(t)$ is the probability density function. The expectation value for a discrete variable means the sum of the probability of each possible outcome multiplied by the outcome value. ${ }^{46}$ For an inherently continuous variable and equally likely domain assumption, $P(t)=\frac{1}{\tau}$, where $\tau$ is the range of the independent variable $t$. Assuming $\tau=N \Delta t$, where $\Delta t$ is the time interval, $E$ can be computed for discrete values as follows:

$$
E[x(t)]=\frac{1}{N} \sum_{i=1}^{N} x_{i},
$$

which is the arithmetic mean. The auto- and cross-spectral densities are then defined as follows:

$$
\begin{aligned}
& G_{x x}(f)=E[X(\omega) X(\omega)], \\
& G_{x y}(f)=E[X(\omega) Y(\omega)] .
\end{aligned}
$$

The capital letters in Eq. (22) denote Fourier transform functions.

The numerical value of coherence is always between 0 and 1. Zero coherence means that the two signals are completely unrelated, while unity implies that they are fully linearly related. It is important to note that the numerical value of the coherence function is not a direct measure of the extent of dissipation mechanisms. In the current study, the coherence function is calculated between the wave at the inlet and another location in the duct. Coherence analyses are also conducted for different points on a single wave front. The coherence analyses are presented using Strouhal numbers on the basis of the flow bulk velocity and length or width of the channel. These are

$$
\begin{aligned}
& S t_{1}=\frac{f(H z)}{\frac{U_{b}}{L}}, \\
& S t_{2}=\frac{f(H z)}{\frac{U_{b}}{2 d}} .
\end{aligned}
$$

\section{E. Wave front detection}

In Sec. III, a criterion is needed to identify the wave front and rear. Approaching the wave in any cross section of the channel, the temperature of the base flow rises and then reaches a peak value and subsequently decreases. Due to the interactions between the wave and the base flow, finding the precise location of the wave rear could be a challenge. In the current study, the wave rear is identified by

$$
\frac{T_{a v e}-\bar{T}_{a v e, i}}{\bar{T}_{a v e, i}}=0.02,
$$

in which $T_{\text {ave }}$ is the mass-weighted average temperature of the flow in an arbitrary cross section of the channel and $\bar{T}_{a v e, i}$ is the flow temperature prior to the release of the entropy wave. The wave front is then found by an infinitesimal increment in the flow temperature. This is done by setting the right-hand side of Eq. (24) equal to 0.001. It is finally noted that the massweighted average for a scalar variable $\phi$ is defined as $\frac{\int \phi \rho|\vec{v} \cdot d \vec{A}|}{\int \rho|\vec{v} \cdot d \vec{A}|}$, where $\vec{v}$ and $\vec{A}$ are the velocity and area vectors, respectively.

\section{RESULTS AND DISCUSSION}

In a turbulent flow, entropy waves are subject to dissipation and dispersion. This is due to turbulent mixing, heat transfer, and non-uniformities in the flow field. For the configuration shown in Fig. 1, the initial amplitude of the temperature disturbance, Reynolds number (Re), turbulence intensity, and thermal boundary condition on the channel walls may influence the decay of entropy waves. In the current work, the initial amplitude of the wave and Reynolds number are kept constant, while other parameters are varied. The channel walls (see Fig. 1) can either include convective heat transfer with the surroundings or be adiabatic. The former is a rarely investigated and more realistic thermal boundary condition in gas turbine combustors and the latter has been used in the previous studies of entropy waves. ${ }^{28}$ Table I provides a list of the selected test cases.

\section{A. General behavior of the wave}

In this section, the wave dissipation and dispersion broadly correspond to the decrease in the amplitude of the entropy wave and wave deformation, respectively. More rigorous definitions of dissipation and dispersion will be put forward later. Figure 6 shows the snapshot of $\Delta T / T_{\text {base flow }}$ for the wave advection through the channel. The left column of this figure corresponds to the early stages of wave advection, while on the right column, the wave has already travelled most of the channel length. This figure provides a clear visual demonstration of the dissipation and dispersion processes. A slower flow in the vicinity of the walls results in a noticeable dispersion of the wave in this region. Further, the pronounced wave dissipation and dispersion in cases with convective heat transfer on the walls are clear [see Figs. 6(c) and 6(d)]. A comparison between Figs. 6(a) and 6(b) and also Figs. 6(c) and 6(d) reveals that higher turbulence intensity has, generally, resulted in intensifying the wave dispersion. This is found by noting the thinner hot core of the wave at the outlet in cases with higher turbulence intensity. The snapshots also show that as a consequence of wave decay, heat diffuses into the base

TABLE I. The investigated test cases.

\begin{tabular}{lccc}
\hline $\begin{array}{l}\text { Thermal boundary } \\
\text { condition on the external } \\
\text { surface of the channel }\end{array}$ & $\begin{array}{c}\text { Reynolds } \\
\text { number }\end{array}$ & $\begin{array}{l}\text { Turbulence intensity } \\
\text { of the inlet flow (\%) }\end{array}$ & Case number \\
\hline Adiabatic & 13000 & 5 & 1 \\
Adiabatic & 13000 & 20 & 2 \\
Convective & 13000 & 5 & 3 \\
Convective & 13000 & 20 & 4 \\
\hline
\end{tabular}




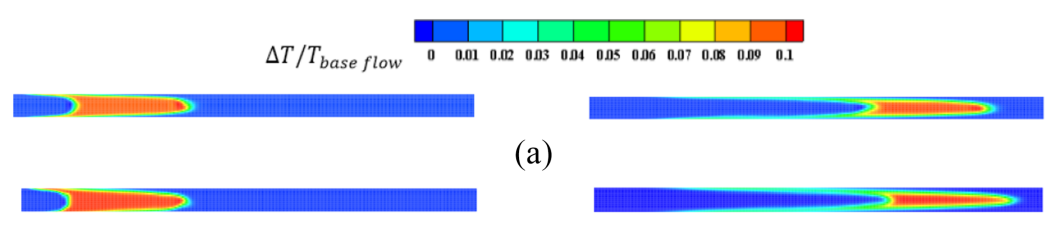

(b)

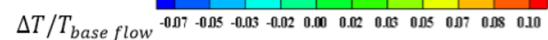

(c)

(d)
FIG. 6. Snapshots of $\Delta T / T_{\text {base flow }}$ for the advection of the wave along the channel: (a) case 1, (b) case 2, (c) case 3 , and (d) case 4 . flow and thus a larger volume of the flow becomes thermally affected.

To further illustrate the dissipation and dispersion processes, Figs. 7 and 8 depict the mass-weighted average variation of $\Delta T / T_{\text {baseflow }}$ during the wave advection. In these figures, the non-dimensional time is defined as time/ $\left(L / U_{b}\right)$, in which $L$ and $U_{b}$ are the channel length and bulk flow velocity, respectively. Figures 7 and 8 clearly show a considerable loss of the wave amplitude (wave dissipation) in all investigated cases. It is also evident in these figures that by increasing turbulence intensity, the flow imparts stronger decaying effects on the entropy waves. As shown in Fig. 8, heat losses by convective heat transfer on the walls cause a reduction in the base flow temperature. This strengthens the tendency of heat transfer from the entropy waves to the base flow and therefore intensifies the wave dissipation.
Figures 7 and 8 show that the dissipation of the wave involves a decay of its low density (high temperature) part. This general trend has been quantified in Fig. 9. The surface area underneath the waves in Figs. 7 and 8 is a representation of the sensible enthalpy of the thermal disturbance. This surface area could be divided into four quarters corresponding to the magnitudes of $\frac{\Delta T}{T_{\text {basefow }}}$. In such a division, that part of the wave surface area with mass-weighted average of $\Delta T / T_{\text {base flow }}$ between 0.075 and 0.1 is referred to as the top quartile. Accordingly, the fraction of the wave surface area with mass-weighted average of $\Delta T / T_{\text {base flow }}$ between 0.025 and 0 is regarded as the bottom quartile. Figure 9 shows the ratios of the top and bottom quartiles to the total surface area of the wave as it travels along the channel. During advection of the wave, energy is subtracted from the top quartile and thermal energy is added to the bottom quartile. Hence, the ratio of the top quartile to the total wave surface becomes nearly zero closer to the channel outlet and (a)

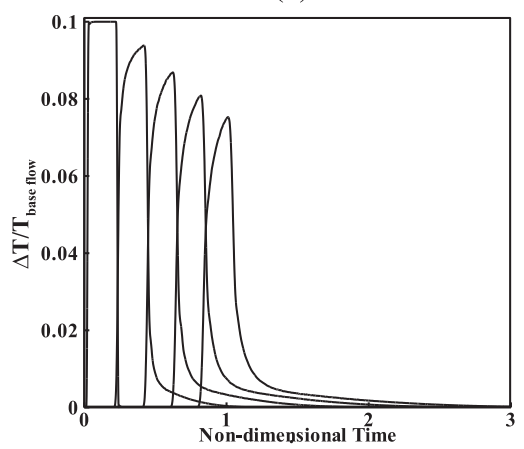

(a)

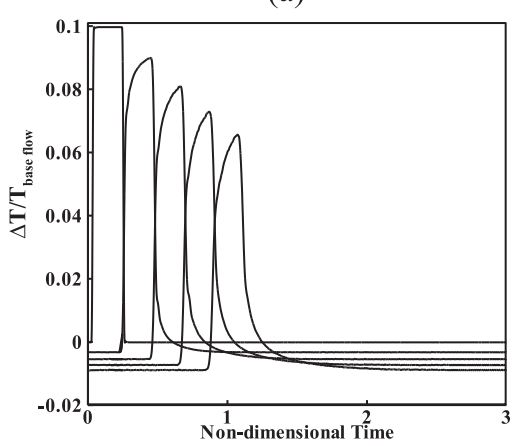

(b)

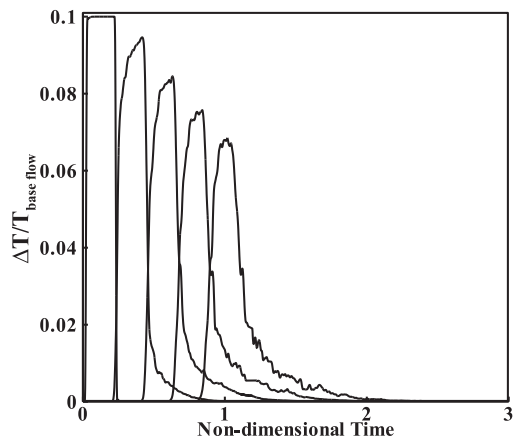

(b)

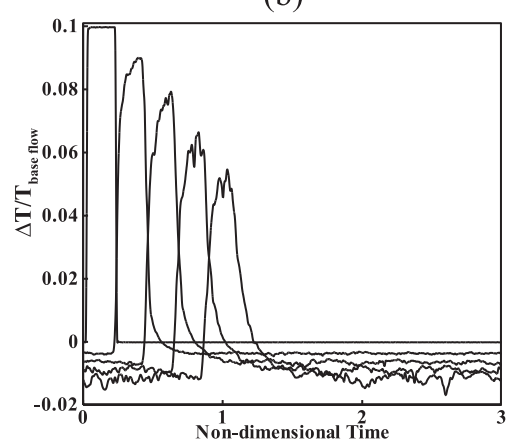

FIG. 7. Variation of the mass-weighted average $\Delta T / T_{\text {base flow }}$ during advection of the entropy wave in the adiabatic channel for (a) case 1 and (b) case 2 .
FIG. 8. Variation of the mass-weighted average $\Delta T / T_{\text {baseflow }}$ during advection of the entropy wave in the heat transferring channel for (a) case 3 and (b) case 4 . 
(a)

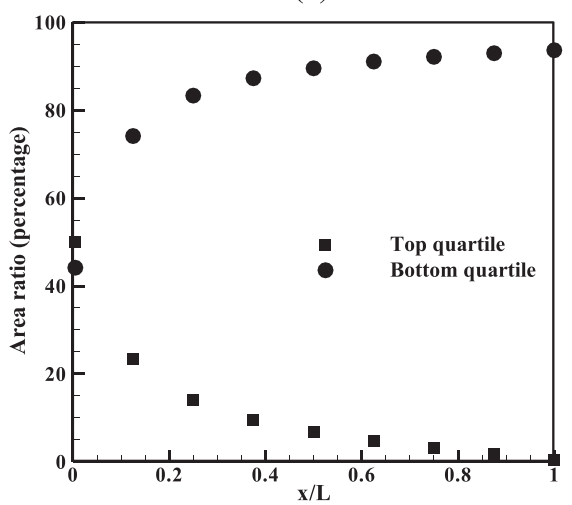

(c)

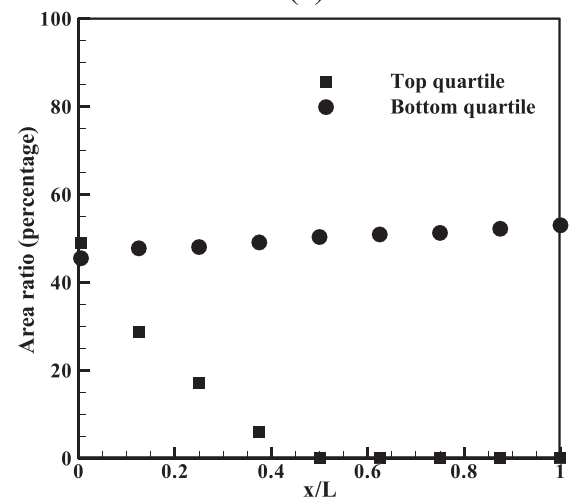

(b)

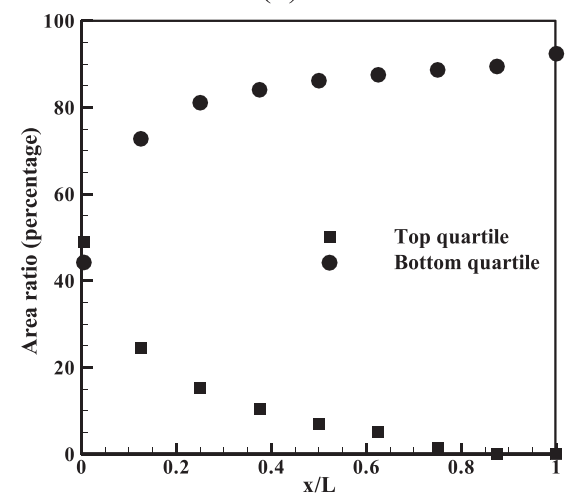

(d)

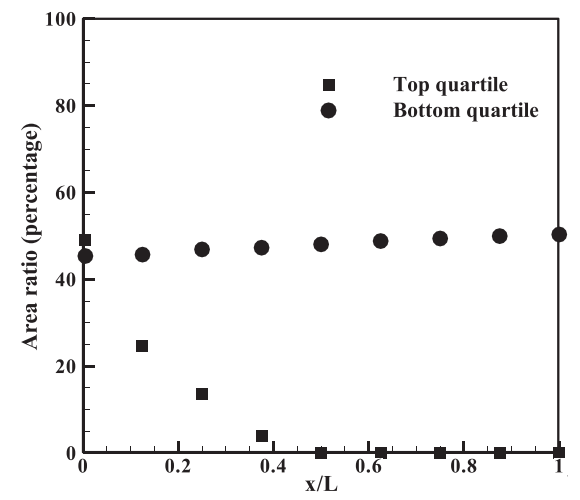

FIG. 9. Ratio of the top and bottom quartiles to the total surface area of the wave calculated for mass-weighted average temperature: (a) case 1 , (b) case 2, (c) case 3 , (d) case 4 . that of the bottom quartile grows in numerical value. A comparison between Figs. 9(a) and 9(b) and also Figs. 9(c) and 9(d) shows that this trend remains mostly indifferent to the changes in turbulence intensity. However, it is majorly influenced by the modifications in the thermal boundary condition. Figures 9(c) and 9(d) show that in the heat transferring cases, the top quartile ratio quickly falls to nearly zero. Interestingly and in sharp distinction to that observed in the adiabatic cases [Figs. 9(a) and 9(b)], the bottom quartile ratio in these figures never takes values greater than $60 \%$. This means that in heat transferring cases, the total thermal energy (sensible enthalpy) of the wave is quite uniformly distributed amongst the whole range of the temperature difference. However, in adiabatic cases, most of the enthalpy is transferred to the bottom quartile.

It is noted that Figs. 7 and 8 are qualitatively similar to those of Morgans et al. ${ }^{28}$ However, unlike the current discussion, these authors argued that there is little dissipation of the wave. In explaining this difference, it should be noted that Morgans et al. ${ }^{28}$ defined dissipation on the basis of the total thermal energy of the wave. Hence, the diffusion of heat to the surrounding fluid and the drop of the temperature difference between the wave and the base flow were effectively ignored in their definition of wave dissipation. Clearly, this is distinctive to the approach taken in Fig. 9 (and later in Sec. III C), in which the temperature difference between the wave and the base flow is the main criterion to evaluate the dissipation rate. In fact, here the exergy loss of the wave, represented by the temperature drop, is considered as the basis of the wave dissipation. This is justified by noting that the generation of sound by the acceleration of entropy waves is a manifestation of the conversion of thermal to mechanical energy. A hotter gas with higher sensible exergy has more potential for conversion into mechanical energy or sound. Thus, the loss of the high temperature fraction of the wave reflects the dissipation of sound generation potential.

\section{B. Coherence of entropy waves}

Figure 10 shows coherence of the mass-weighted average of the temperature signal calculated between the inlet and middle of the channel [cross sections $A$ and $C$ in Fig. 1(a)] and also the outlet (cross section $D$ ). Although not shown here, for both cases 1 and 2 , the value of the coherence function calculated between the inlet and cross section $B$ is always one. This is in keeping with the physical intuition, which requires the wave to maintain its coherence in advection over a short distance. Figures 10(a) and 10(b) show that at low turbulence intensity (case 1), the coherence functions calculated between the inlet and middle and also outlet of the channel take values very close to unity. This is an indication of the survival of the entropy wave in the whole channel. It should be, however, noted that as depicted in Fig. 7, the amplitude of the wave drops during the advection process. Yet, for case 1 and on a mass-weighted average basis, the wave remains coherent as it travels along the channel.

This behavior changes significantly in case 2 , in which the turbulence intensity is considerably higher than that in case 1 . Figure 10(c) shows that for case 2, the coherence function calculated between the inlet and middle of the channel is close to one at low frequencies. However, it sharply drops at higher frequencies and reaches almost zero at Strouhal numbers greater than 25. Figure 10(d) shows that extending the analysis to 
(a)

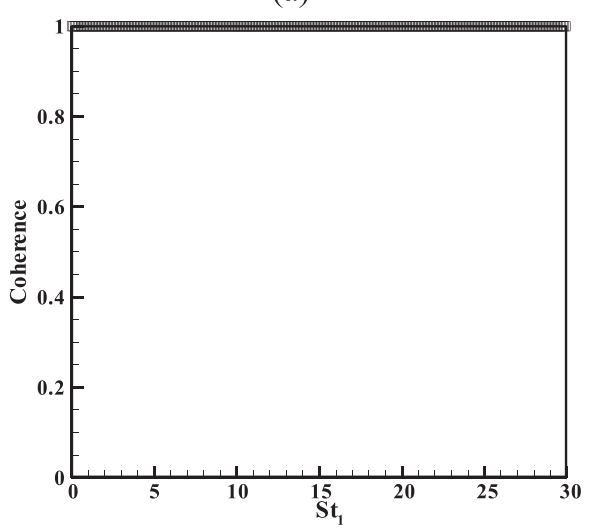

(c)

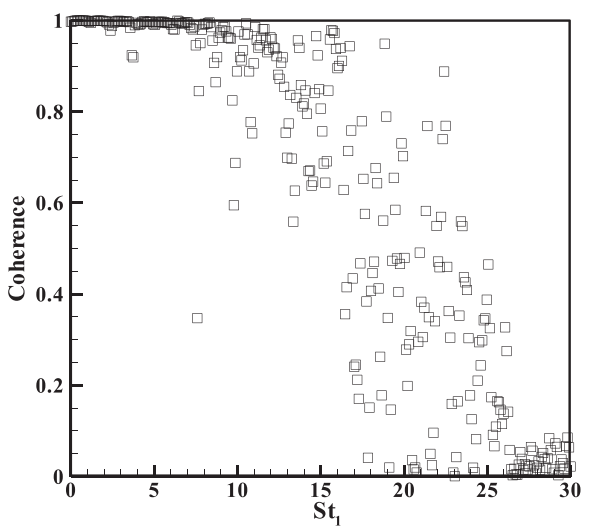

(b)

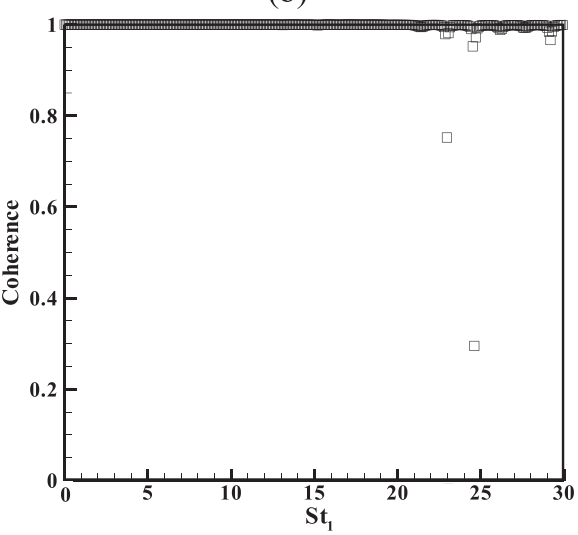

(d)

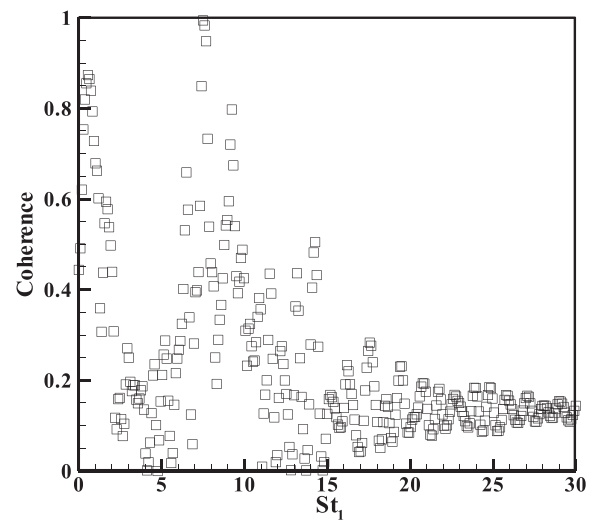

FIG. 10. Coherence function for the mass-weighted average temperature: (a) case 1 , cross sections $A$ and $C$, (b) case 1 , cross sections $A$ and $D$, (c) case 2, cross sections $A$ and $C$, (d) case 2 , cross sections $A$ and $D$. the inlet and outlet of the channel limits the high coherence range to narrow frequency bands at the beginning and middle of the spectrum. This figure indicates that by the time the entropy wave reaches the outlet of the duct it has largely lost its coherence with respect to the corresponding wave at the inlet.

Addition of convective heat transfer on the external walls of the duct, in cases 3 and 4 , results in major modifications in the wave coherence. These are illustrated in Fig. 11 showing a general drop of coherence in cases 3 and 4 compared with cases 1 and 2, shown in Fig. 10. Figure 11(a) depicts the coherence function calculated for case 3 between the inlet and one eighth of the channel length [cross section $B$ in Fig. 1(a)]. This figure features a broad similarity with Fig. 10(c), in which the value of coherence is one at low frequencies and drops at higher frequencies and eventually becomes zero. Unlike Fig. 10(c), however, the decline of coherence in Fig. 11(a) is not monotonic and there are some increases in the value of coherence in the middle of the spectrum before a sudden drop to zero. Figure 11(c) shows that by increasing the turbulence intensity (case 4), the entropy wave has mostly lost its coherence resulting in a scattered graph. Calculation of the coherence between the inlet and outlet of the channel for cases 3 and 4 [Figs. 11(b) and 11(d)] results in a nearly zero value for the entire spectrum. This implies that regardless of the level of turbulence intensity, the entropy wave loses its entire coherence during advection in the heat transferring channel. The strong influence of heat transfer on the decay of the wave is somehow expected. The thermal non-uniformity caused by the convective cooling of the channel intensifies the potential for heat transfer from the wave and therefore accelerates the decay process. This is analogous to flow non-uniformity effects on the decay of entropy waves discussed by Morgans et al. ${ }^{28}$ However, it appears that the thermal non-uniformities have a stronger destructive effect on the wave than those induced by the flow shear stress. Interestingly, the results shown in Fig. 11 indicate that under convective cooling, even low turbulence intensities suffice for large annihilation of the entropy wave.

The analyses presented so far were concerned with the mass averaged signals and hence excluded the spatial coherence on the wave surface. In reality, the annihilating mechanisms can destroy the spatial coherence of the wave. Evaluating the extent of such destruction is of significance, as it determines the validity range of one-dimensional theories commonly used for modeling the conversion of entropy waves into sound, for instance, Refs. 12-14. Figure 12 shows the coherence function calculated for cases 1 and 2 between points 1 and 2 and also points 1 and 3 in Fig. 1(b). The calculations were performed at the middle of the channel (cross section $C$ ). This figure shows that for low turbulence intensity at the inlet, the points on the wave front demonstrate a perfect coherence [see Figs. 12(a) and 12(b)]. Increasing the turbulence intensity seems to have a minor effect on the spatial coherence of the wave front. Figures 12(c) and 12(d) show that enhancing turbulence intensity in case 2 has slightly decreased the value of the coherence function at the high frequency end of the spectrum, while otherwise coherence is close to one. This behavior is different to that observed in Fig. 10(c), in which the coherence of case 2, 
(a)

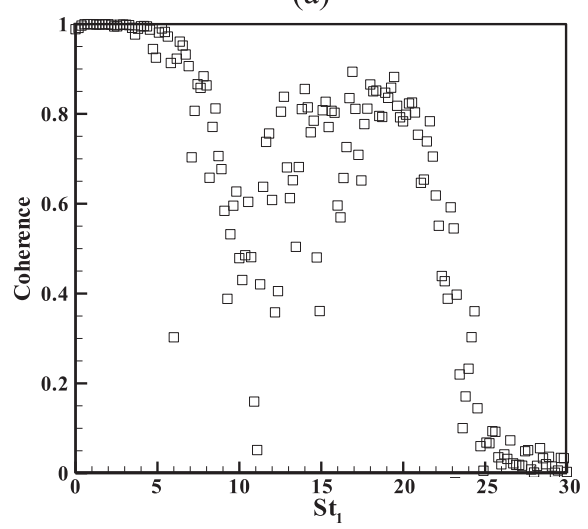

(c)

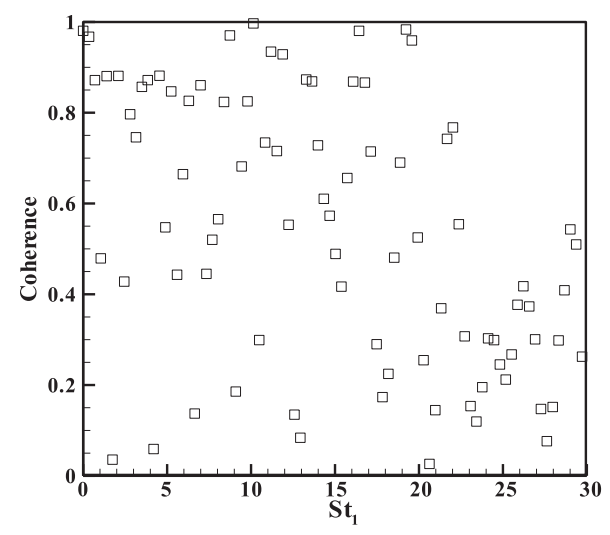

(a)

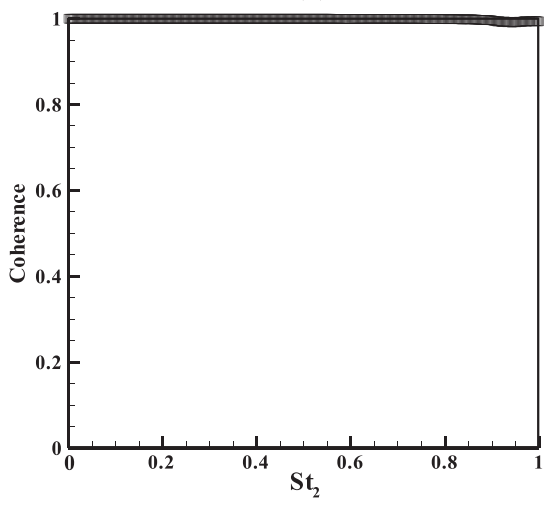

(c)

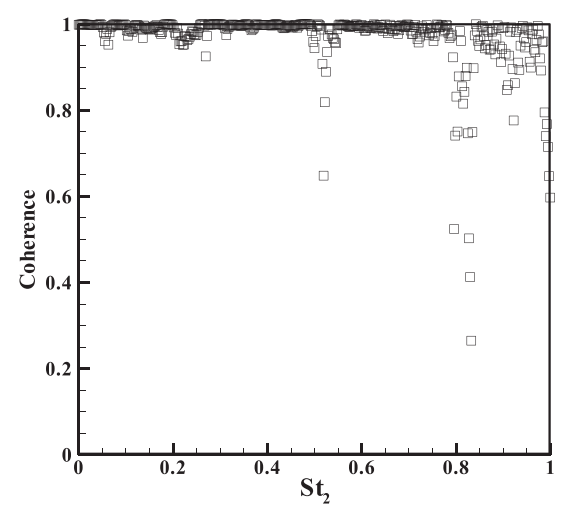

(b)

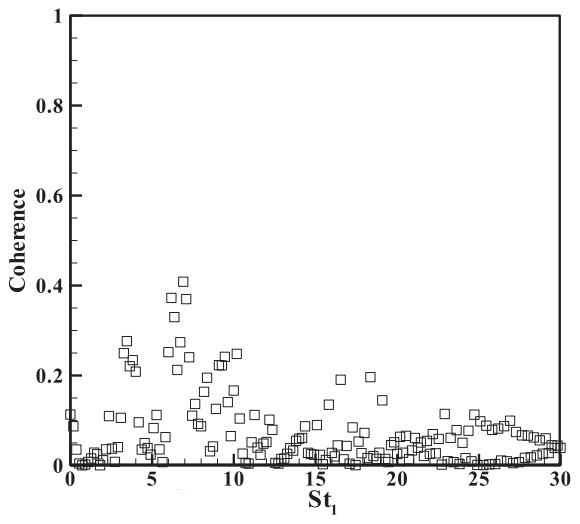

(d)

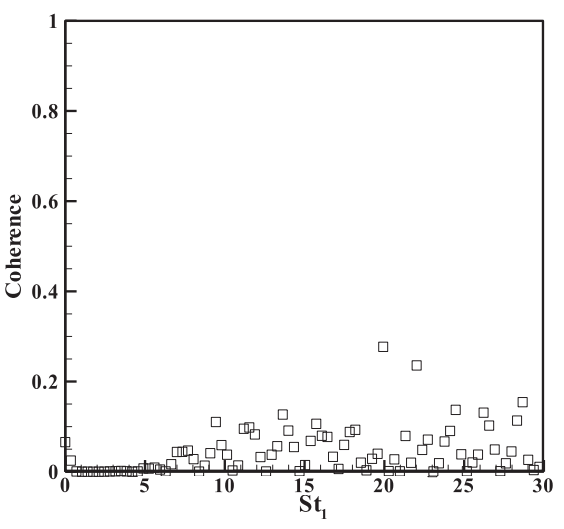

(b)

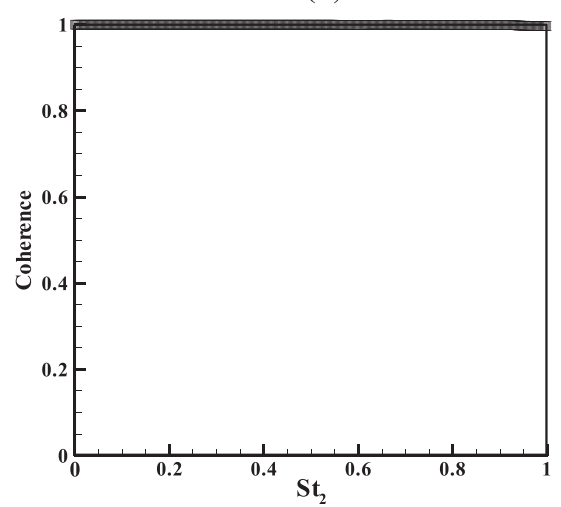

(d)

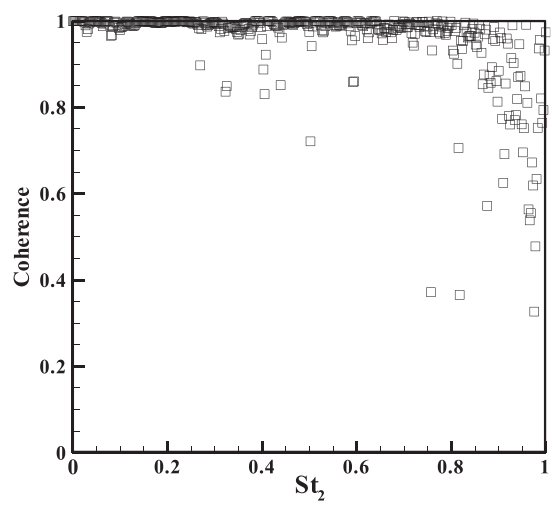

FIG. 11. Coherence function for massweighted average temperature: (a) case 3 , cross sections $A$ and $B$, (b) case 3 , cross sections $A$ and $D$, (c) case 4, cross sections $A$ and $B$, (d) case 4 , cross sections $A$ and $D$.
FIG. 12. Coherence function for the temperature at the channel half-length (cross section $C$ ) between (a) case 1, points 1 and 2, (b) case 1 points 1 and 3 , (c) case 2, points 1 and 2, and (d) case 2 points 1 and 3 . 
(a)

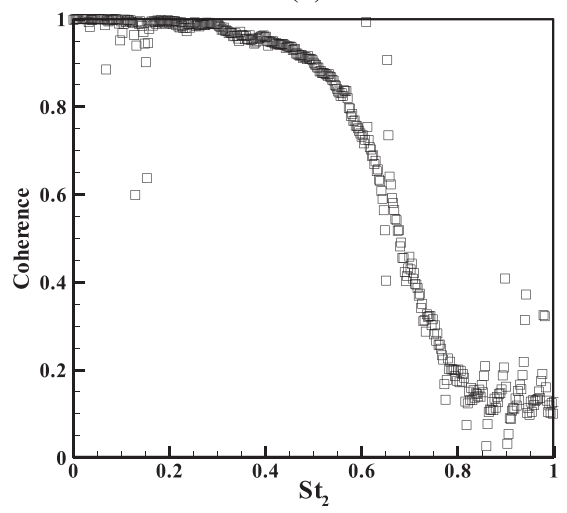

(c)

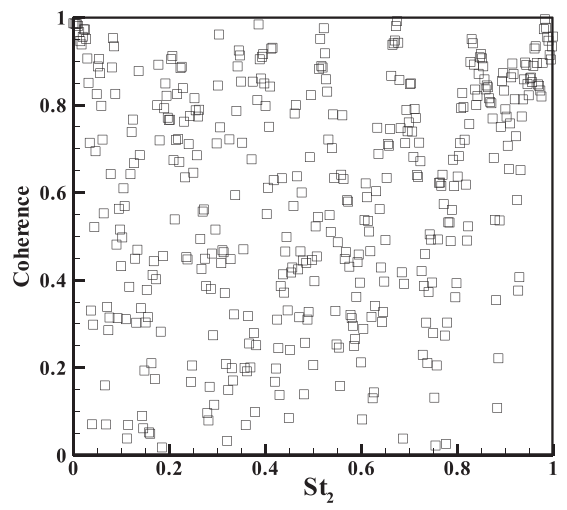

(b)

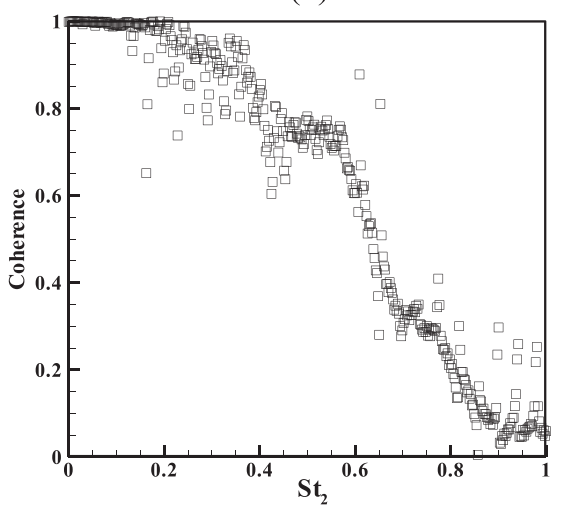

(d)

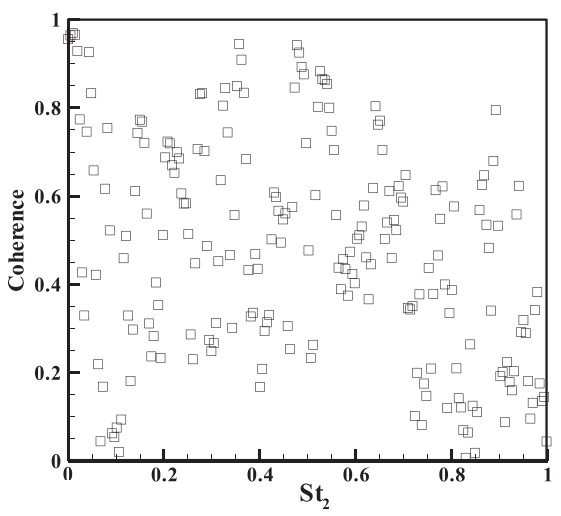

FIG. 13. Coherence function for the temperature at the outlet (cross section $D$ ) between (a) case 1 and points 1 and 2, (b) case 1 and points 1 and 3, (c) case 2 and points 1 and 2, and (d) case 2 and points 1 and 3 . calculated on mass-weighted average basis and between cross sections $A$ and $C$, is predominately below one.

In Fig. 13 the analyses presented in Fig. 12 are repeated at cross section $D$ or outlet of the channel. It is clear from parts (a) and (b) of this figure that for case 1 and at low frequencies, points 1 and 2 and also points 1 and 3 in Fig. 1(b) are highly correlated. As the frequency increases, the numerical value of the coherence function decreases in an almost monotonic way and ultimately settles at around 0.1 . This trend implies that in case 1 , low frequency (high wavelength) components of the wave can very well survive the flow. At higher dimensionless frequencies, the wave becomes much more vulnerable to the flow annihilation mechanisms and the high frequency components are essentially destroyed. In practice, entropy waves often generate a low frequency noise ${ }^{11}$ Figs. 13(a) and 13(b) and also Figs. 10(c) and 10(d) provide an explanation for this behavior. Turbulent mixing quickly annihilates any small-scale flow non-uniformity or high frequency component of entropy waves. Larger non-uniformities or low frequency components of the wave need a longer advection time to decay and therefore find a better chance to travel the entire length of the channel and reach the exit nozzle. By intensifying the turbulence level, the density non-uniformities are annihilated more rapidly and therefore even large wavelength components of the wave are influenced. This could result in the loss of coherence between different points on the wave as shown in Figs. 13(c) and 13(d). As already illustrated in Figs. 10 and 11, the inclusion of convective heat transfer further strengthens the decay of entropy waves. Regardless of the turbulence intensity and even at the middle of the channel, this leads to the generation of a scattered coherence graph with an average value close to zero. It follows that in heat transferring cases 3 and 4, the spatial coherence of entropy waves is almost completely destroyed within the first half of the channel. For brevity reasons, these results are not shown here.

\section{Dissipation and dispersion of the wave}

Dissipation of a mechanical wave implies a decrement in the wave energy. ${ }^{47}$ For an entropy wave, the amplitude of the temperature disturbance represents the wave energy. Thus, for the configuration shown in Fig. 1 and in the frequency domain, a quantitative measure of dissipation can be devised as follows:

$$
\operatorname{Dissipation}\left(\omega_{a}\right)=\left.\frac{\mathcal{F}[T(x=0.05 L)]-\mathcal{F}\left[T\left(x_{a}\right)\right]}{\mathcal{F}[T(x=0.05 L)]}\right|_{\omega=\omega_{a}}
$$

where $\mathcal{F}$ is the discrete Fourier transform. Further, in Eq. (25), $x_{a}$ is the longitude position of an arbitrary cross section of the channel in Fig. 1(a) and $\omega_{a}$ is an arbitrary frequency. Here, the value of dissipation $\times 100$ is regarded as the dissipation index. Clearly, for a non-dissipative wave, the Fourier components of the wave do not change through the advection process, and therefore the dissipation index will be zero for all values of frequency. Conversely, for a highly dissipative wave, Fourier components diminish in magnitude, and thus the dissipation index takes values close to $100 \%$. As an essential point, it should be emphasized that Eq. (25) does not make any assumption about the linearity of the system under 
(a)

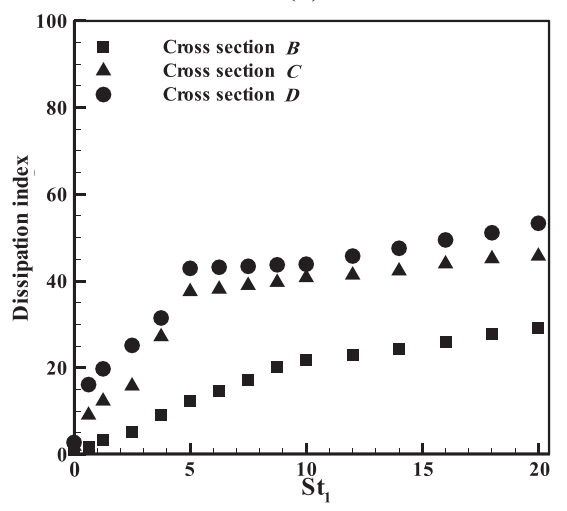

(c)

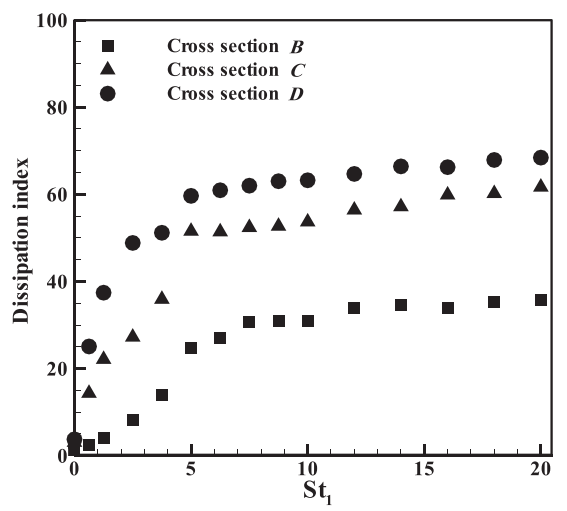

(b)

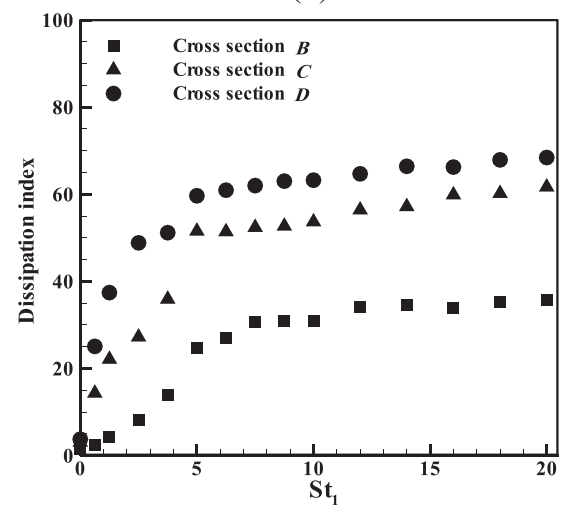

(d)

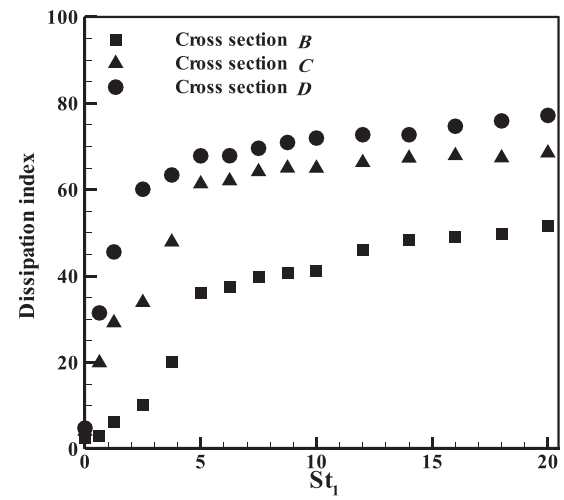

FIG. 14. Mass-weighted averaged dissipation index versus non-dimensional frequency for (a) case 1, (b) case 2, (c) case 3 , (d) case 4 . investigation. However, the coherence analysis presented in Sec. III B is limited to linear systems. ${ }^{45}$

Figure 14 shows the results of calculating the dissipation index at three different cross sections along the channel. In this figure, the mass-weighted average of the temperature signals of cases 1 to 4 is analyzed. In keeping with the earlier results, Fig. 14 confirms that for all investigated test cases and locations along the channel, dissipation is negligibly small at very low frequencies. Yet, it increases significantly at higher frequencies. The rate of increase of the dissipation index is particularly high for Strouhal numbers up to about 5. As expected, in all cases and for a given frequency, the dissipation index grows in magnitude with the distance from the channel inlet. Further, test cases with external heat transfer (cases 3 and 4 ) and higher turbulence intensity (cases 2 and 4 ) feature higher values of the dissipation index. It is also inferred from Fig. 14 that for all frequencies, the dissipation of the wave in the first half of the channel is stronger than that in the second half. This is such that in all test cases investigated in Fig. 14, there is a significant difference between the value of dissipation indices calculated at cross sections $B$ and $C$. However, the difference between the indices evaluated at cross sections $C$ and $D$ (middle and exit of the channel) is relatively small. Given that the dissipation mechanisms are applied equally to the wave through the entire channel, this behavior represents the effect of the initial amplitude. Within the first half of the channel, the temperature disturbance is still of high amplitude and has a large potential to diffuse into the base flow. This tendency decays in the second half of the channel and results in a slower rate of wave dissipation.
The mass-weighted average analysis shown in Fig. 14 excludes the variations of the dissipation index on the surface of the wave. By considering points 1, 2, and 3 in Fig. 1(b), this effect has been investigated in Figs. 15 and 16. Although the qualitative trends observed in Fig. 15 (cases 1 and 2) and Fig. 16 (cases 3 and 4) are similar to those of Fig. 14, there exist some disparities. First, in some cases, the distribution of dissipation indices is more uniform in comparison to the massweighted average case shown in Fig. 14. This is particularly noticeable in Figs. 15(a) and 15(b) and also Figs. 16(b) and 16(c). In these figures, for a fixed frequency, the calculated dissipation indices at different cross sections show comparable differences with each other. This indicates that although the mass averaged dissipation is stronger in the first half of the channel, some points along the wave could be continuously dissipated with a nearly constant rate. Second, the value of the local dissipation index can be noticeably different to that of the mass-weighted average for the same case. For instance, Fig. 15(c) shows that in case 2, the high frequency dissipation index could reach only 50\% when calculated at point 1 . However, the corresponding average dissipation index in Fig. 14(b) takes the value of 70\%. Similar arguments can be made about other subfigures in Figs. 15 and 16. Further, a comparison between Figs. 14(b) and 14(c) and also Figs. 16(b) and 16(c) clearly indicates that convective cooling of the channel is a very strong dissipation agent, which can surpass the turbulence intensity effects.

Decaying advective-diffusive waves can feature wave stretch and deformation. Entropy waves at the channel inlet have a narrow square shape. In the course of advection through 
(a)

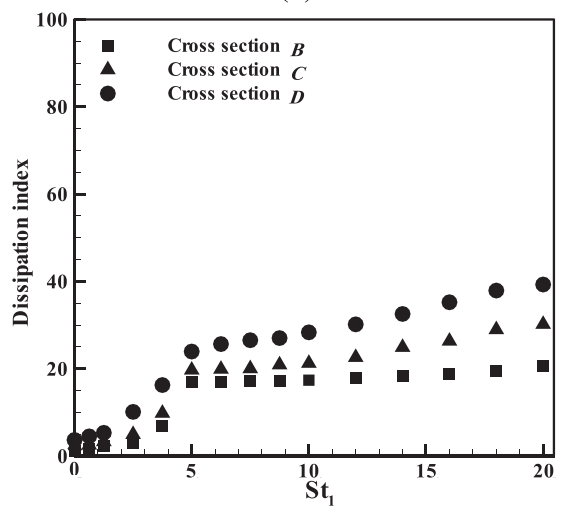

(c)

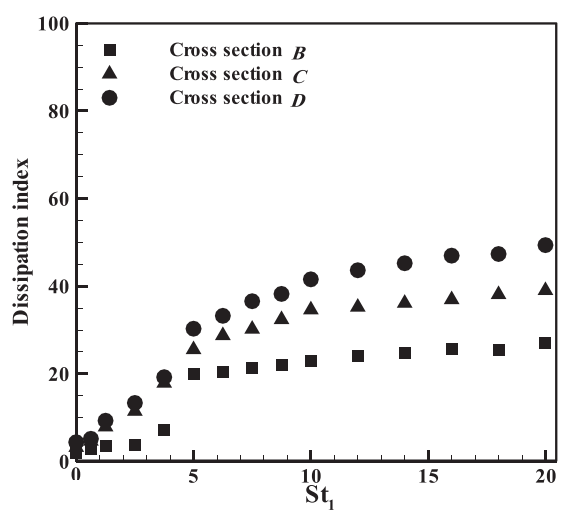

(a)

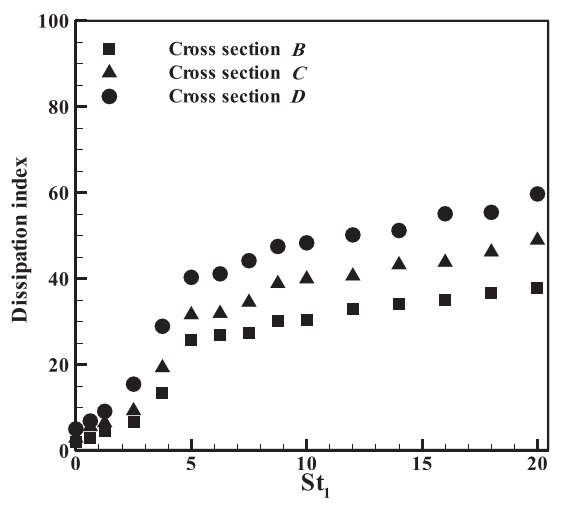

(c)

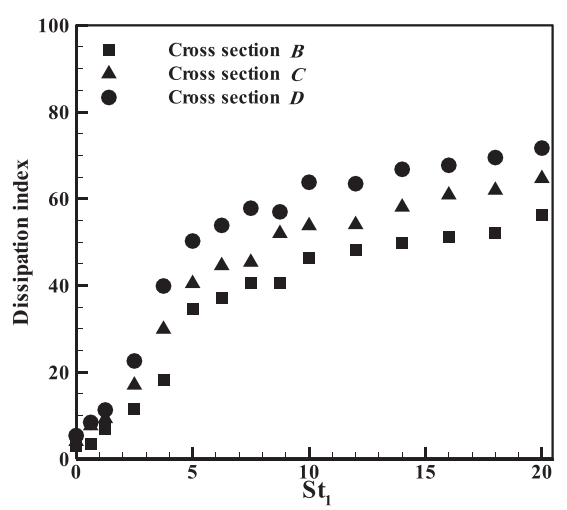

(b)

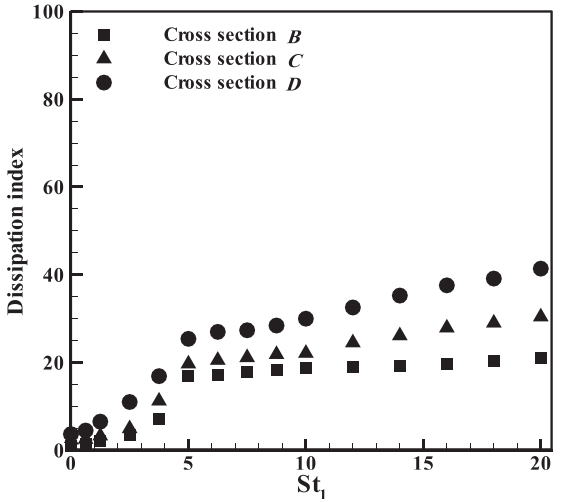

(d)

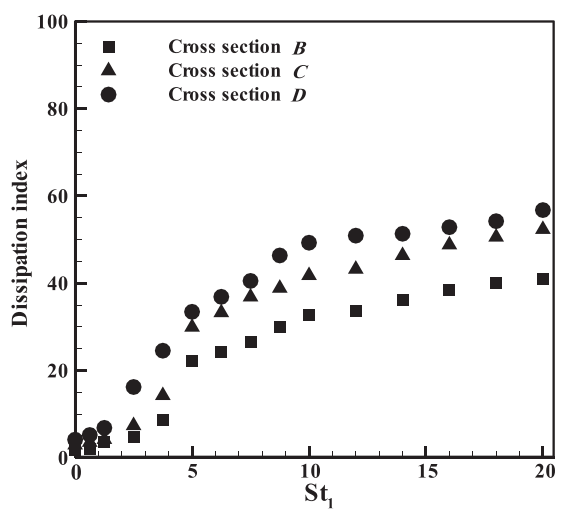

(b)

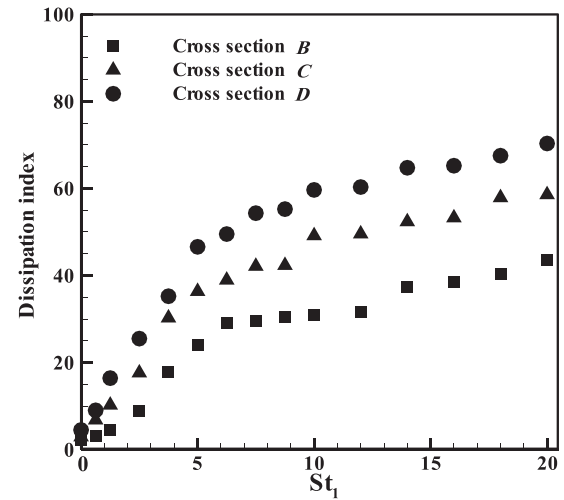

(d)

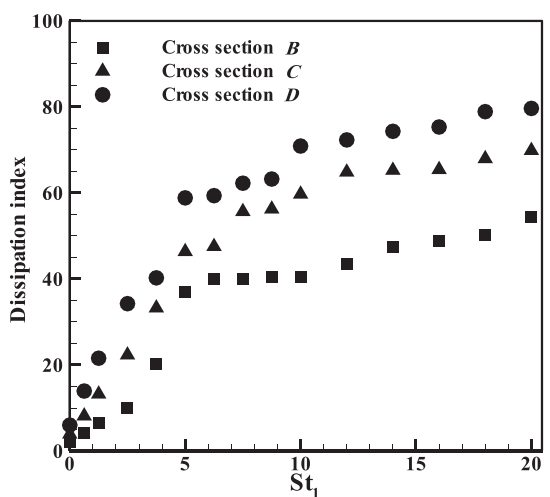

FIG. 15. Dissipation index versus nondimensional frequency for (a) case 1 at point 1 , (b) case 1 at point 2 , (c) case 2 at point $1,(\mathrm{~d})$ case 2 at point 2 .
FIG. 16. Dissipation index versus nondimensional frequency for (a) case 3 at point 1 , (b) case 3 at point 2, (c) case 4 at point $1,(\mathrm{~d})$ case 4 at point 2 . 
the channel, they experience significant deformations (see Figs. 7 and 8). This reshaping process modifies the topology of the wave spectrum such that some spectral components are effectively canceled and new components appear. The net effect is a destruction of the initial wave front and hence it could be considered as a manifestation of wave dispersion. Consequently, a dispersion index is defined on the basis of reshaping of the wave spectrum by the flow annihilating mechanisms. The spectral shifts of the first three maxima form a dispersion index, defined as

$$
\text { Dispersion index }=\left(\frac{\Delta \mathrm{f}_{1}}{\mathrm{f}_{1}}+\frac{\Delta \mathrm{f}_{2}}{\mathrm{f}_{2}}+\frac{\Delta \mathrm{f}_{3}}{\mathrm{f}_{3}}\right) \times 100,
$$

where digits 1 to 3 refer to the relative maxima in Fig. 17. This figure shows the spectrum of the entropy wave for case 1 at the entrance of the duct, where it has not been dispersed yet. It further includes the spectrum of the same front close to the exit point of the duct. A very noticeable change in the spectrum is evident, and the spectral shifts of the local maxima are used by Eq. (26) to define the dispersion index.

Figure 18 illustrates the dispersion indices for cases 1 to 4 calculated at points 1,2 , and 3 on three different cross sections of the channel. The dispersion indices have been further calculated for the mass-weighted average signal. This figure shows a clear trend, in which dispersion indices have small numerical values near the channel entrance and grow in magnitude as the exit point is approached. The extent of this growth varies significantly amongst the four investigated cases. As expected, case 1 [Fig. 18(a)] has the lowest growth of the dispersion index. Intensifying the inlet turbulence level in case 2

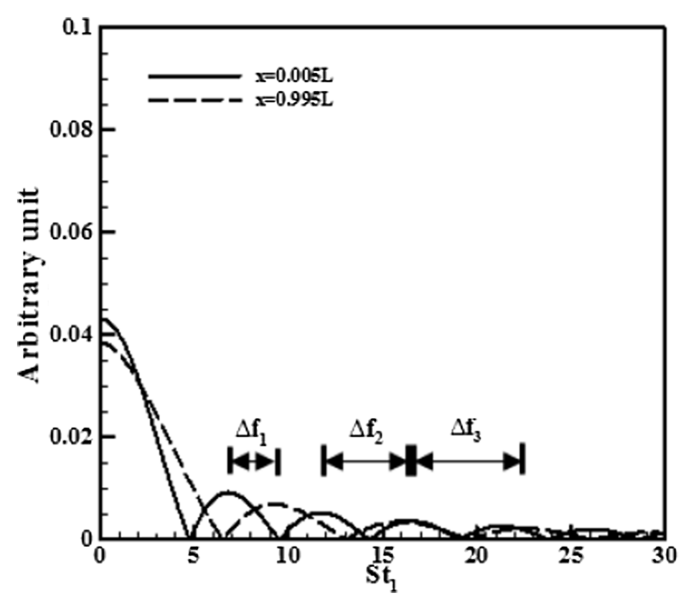

FIG. 17. Illustration of frequency shift in the spectrum of an entropy wave at the inlet and outlet of the channel (case 1).

[Fig. 18(b)] or addition of convective heat transfer in case 3 [Fig. 18(c)] increases this growth significantly. Nonetheless, similar to that discussed with regard to wave dissipation, the heat transfer effects on the wave dispersion appear to be stronger than those of turbulence intensity. Case 4 features high turbulence intensity and convective cooling and has the highest value of the dispersion index amongst the investigated cases. It is also noted that in cases 3 and 4 , there is no considerable difference among the dispersion indices calculated at points 1,2, and 3 and that of mass-weighted average. Yet, this is not the case in Figs. 18(a) and 18(b) (cases 1 and 2). Once (a)

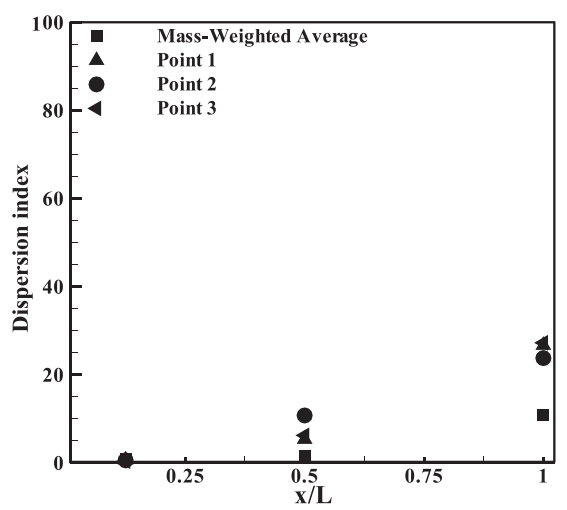

(c)

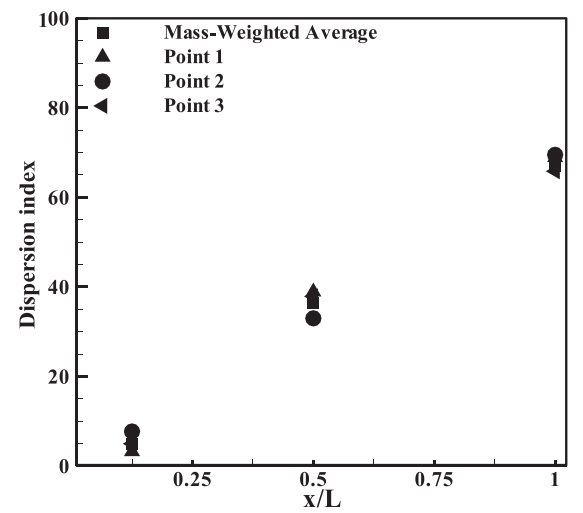

(b)

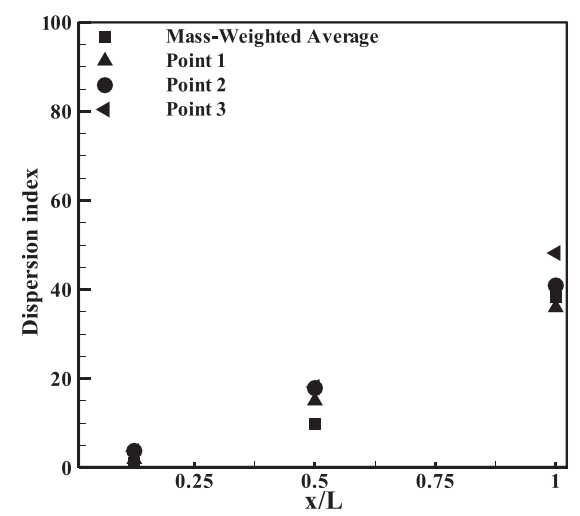

(d)

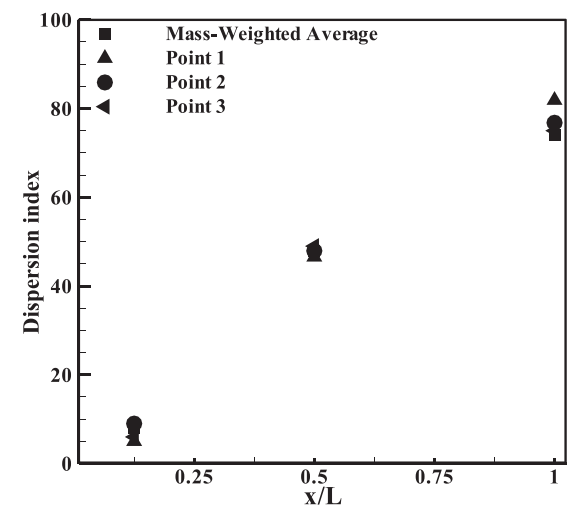

FIG. 18. Dispersion index between the channel inlet and sections of $x=0.125 L, x=0.5 L$, and $x=0.995 L$ for temperature variation at points 1 to 3 and mass-weighted average: (a) case 1 , (b) case 2 , (c) case 3 , (d) case 4 . 
again, this indicates that the strong dispersion induced by heat transfer dominates any other spatial non-uniformity effects on the wave front.

\section{CONCLUSIONS}

Compressible large eddy simulations were conducted to analyze the advection process of entropy waves in a channel. The external surfaces of the channel could be either convectively cooled or maintained adiabatic, and two different values of turbulence intensity were considered for the incoming flow. Entropy waves were introduced through temperature disturbances at the channel inlet, which were advected downstream by the mean cold flow. The decay of these disturbances was investigated using different linear and nonlinear post-processing methods. Visualization of the advection process indicated that the wave is subject to considerable loss of amplitude, in which sensible enthalpy is deducted from the wave and is added to the adjacent fluid. This dissipates the wave thermal exergy and results in wave deformation or dispersion. Coherence of the wave was subsequently calculated at different cross sections across the channel on the basis of mass-weighted average and also for specific points on the wave front. These analyses showed that for adiabatic channels and low turbulence intensities, entropy waves remain mostly coherent over the entire advection process. However, intensifying the turbulence level or adding convective wall cooling could significantly damage the wave coherence. In the course of the advection process, the high frequency or short wavelength components of the entropy wave are annihilated quickly. The low frequency components have a better chance to survive the flow. Depending on the level of turbulence intensity and thermal boundary conditions, they may reach the end of the channel. This behavior explains the low frequency character of entropy noise in combustion devices. Introduction of non-linear dissipation and dispersion indices confirmed the findings of the coherence analyses. It also revealed that the rate of wave decay is often higher at the first half of the channel, where the wave still has a relatively high amplitude. Heat transfer to the surroundings was identified as a major contributor to the wave dissipation and dispersion process. In heat transferring channels, entropy waves can only persist over relatively short distances. The findings of this paper appear to be in keeping with the experimental observations made in heat transferring combustors of real gas turbines. ${ }^{25}$ They are also in agreement with the pervious simulations in adiabatic flows. 28

${ }^{1}$ J. E. Ffwocs Williams and M. Howe, "The generation of sound by density inhomogeneities in low Mach number nozzle flows," J. Fluid Mech. 70, 605 (1975).

${ }^{2}$ M. Howe, "Contributions to the theory of aerodynamic sound, with application to excess jet noise and the theory of the flute," J. Fluid Mech. 71, 625 (1975).

${ }^{3}$ W. C. Strahle, "Combustion noise," Prog. Energy Combust. Sci. 4(3), 157 (1978).

${ }^{4}$ M. Muthukrishnan, W. C. Strahle, and D. H. Neale, "Separation of hydrodynamic, entropy, and combustion noise in a gas turbine combustor," AIAA J. 16(4), 320 (1978).

${ }^{5}$ W. C. Strahle, "Duality, dilatation, diffusion and dissipation in reacting turbulent flows," Symp. (Int.) Combust. 19(1), 337 (1982).
${ }^{6}$ W. C. Strahle and M. Muthukrishnan, "Correlation of combustor rig sound power data and theoretical basis of results," AIAA J. 18(3), 269 (1980).

${ }^{7}$ N. A. Cumpsty and F. E. Marble, "The interaction of entropy fluctuations with turbine blade rows; a mechanism of turbojet engine noise," Proc. R. Soc. London, Ser. A 357(1690), 323 (1977).

${ }^{8}$ N. A. Cumpsty and F. E. Marble, "Core noise from gas turbine exhausts," J. Sound Vib. 54(2), 297 (1977).

${ }^{9}$ S. Candel, D. Durox, S. Ducriux, A.-L. Birbaud, N. Noiray, and D. Schuller, "Flame dynamics and combustion noise: progress and challenges," Int. J. Aeroacoustics 8(1), 1 (2009).

${ }^{10}$ T. C. Lieuwen, Unsteady Combustor Physics (Cambridge University Press, 2012).

${ }^{11}$ A. S. Morgans and I. Duran, "Entropy noise: A review of theory, progress and challenges," Int. J. Spray Combust. Dyn. 8(4), 285 (2016).

${ }^{12}$ F. E. Marble and S. M. Candel, "Acoustic disturbance from gas nonuniformities convected through a nozzle," J. Sound Vib. 55(2), 225 (1977).

${ }^{13}$ W. H. Moase, M. J. Brear, and C. Manzie, "The forced response of choked nozzles and supersonic diffusers," J. Fluid Mech. 585, 281 (2007).

${ }^{14}$ C. S. Goh and A. S. Morgans, "Phase prediction of the response of choked nozzles to entropy and acoustic disturbances," J. Sound Vib. 330, 5184 (2011).

${ }^{15}$ M. Leyko, S. Moreau, F. Nicoud, and T. Poinsot, "Numerical and analytical modelling of entropy noise in a supersonic nozzle with a shock," J. Sound Vib. 330(16), 3944 (2011).

${ }^{16}$ I. Duran, S. Moreau, and T. Poinsot, "Analytical and numerical study of combustion noise through a subsonic nozzle," AIAA J. 51(1), 42 (2013).

${ }^{17}$ I. Duran and S. Moreau, "Solution of the quasi-one-dimensional linearized Euler equations using flow invariants and the Magnus expansion," J. Fluid Mech. 723, 190 (2013).

${ }^{18}$ M. Huet and A. Giauque, "A nonlinear model for indirect combustion noise through a compact nozzle," J. Fluid Mech. 733, 268 (2014).

${ }^{19}$ F. Bake, C. Richter, B. Mühlbauer, N. Kings, I. Rohle, F. Thiele, and B. Noll, "The entropy wave generator (EWG): a reference case on entropy noise," J. Sound Vib. 326(3), 574 (2009).

${ }^{20}$ F. Bake, N. Kings, and I. Roehle, "Fundamental mechanism of entropy noise in aero-engines: Experimental investigation," J. Eng. Gas Turbines Power 130(1), 011202 (2008).

${ }^{21}$ P. A. Hield, M. J. Brear, and S. H. Jin, "Thermoacoustic limit cycles in a premixed laboratory combustor with open and choked exits," Combust. Flame 156, 1683 (2009).

${ }^{22}$ P. A. Hield and M. J. Brear, "Comparison of open and choked premixed combustor exits during thermoacoustic limit cycle," AIAA J. 46(2), 517 (2008).

${ }^{23}$ C. S. Goh and A. S. Morgans, "The influence of entropy waves on the thermoacoustic stability of a model combustor," Combust. Sci. Technol. 185, 249 (2013)

${ }^{24}$ A. P. Dowling and S. R. Stow, "Acoustic analysis of gas turbine combustors," J. Propul. Power 19(5), 751 (2003).

${ }^{25}$ J. Eckstein, E. Freitag, C. Hirsch, and T. Sattelmayer, "Experimental study on the role of entropy waves in low-frequency oscillations in a RQL combustor," J. Eng. Gas Turbines Power 128(2), 264 (2006).

${ }^{26} \mathrm{~J}$. Eckstein and T. Sattelmayer, "Low-order modeling of low-frequency combustion instabilities in aeroengines," J. Propul. Power 22, 425 (2006).

${ }^{27} \mathrm{~T}$. Sattelmayer, "Influence of the combustor aerodynamics on combustion instabilities from equivalence ratio fluctuations," J. Eng. Gas Turbines Power 125, 11 (2003).

${ }^{28}$ A. S. Morgans, C. S. Goh, and J. A. Dahan, "The dissipation and shear dispersion of entropy waves in combustor thermoacoustics," J. Fluid Mech. 733, R2 (2013).

${ }^{29}$ M. J. Brear, D. C. Carolan, and N. Karimi, "Dynamic response of the exit nozzle of a premixed combustor to pressure and entropic disturbances," in the Proceedings of the 8th Euromech Fluid Mechanics Conference, Bad Reichenhall, Germany, 2010.

${ }^{30} \mathrm{~T}$. Poinsot, "Prediction and control of combustion instabilities in real engines," Proc. Combust. Inst. 36, 1 (2016).

${ }^{31}$ M. Hosseinalipour, A. Fattahi, A. Afshari, and N. Karimi, "On the effects of convecting entropy waves on the combustor hydrodynamics," Appl. Therm. Eng. 110, 901 (2017).

${ }^{32}$ N. Karimi, M. J. Brear, and W. Moase, "Acoustic and disturbance energy analysis of a flow with heat communication," J. Fluid Mech. 597, 67 (2008).

${ }^{33}$ N. Karimi, M. Brear, and W. Moase, "On the interaction of sound with steady heat communicating flows," J. Sound Vib. 329(22), 4705 (2010). 
${ }^{34}$ A. H. Lefebvre, Gas Turbine Combustion (CRC Press, 1998).

${ }^{35}$ F. Nicoud and F. Ducros, "Subgrid-scale stress modelling based on the square of the velocity gradient tensor," Flow, Turbul. Combust. 62(3), 183 (1999).

${ }^{36}$ L. Marcel, O. Metais, and P. Comte, Large-Eddy Simulations of Turbulence (Cambridge University Press, 2005).

${ }^{37}$ J. Smagorinsky, "General circulation experiments with the primitive equations: I. The basic experiment," Mon. Weather Rev. 91(3), 99 (1963).

${ }^{38} \mathrm{X}$. Han and A. S. Morgans, "Simulation of the flame describing function of a turbulent premixed flame using an open-source LES solver," Combust. Flame 162(5), 1778 (2015).

${ }^{39}$ A. Urbano, L. Selle, G. Staffelbach, B. Cuenot, T. Schmitt, S. Ducruix, and S. Candel, "Exploration of combustion instability triggering using Large Eddy Simulation of a multiple injector liquid rocket engine," Combust. Flame 169, 129 (2016).
${ }^{40}$ J. Franke, A. Hellsten, K. H. Schlunzen, and B. Carissimo, "The COST 732 Best Practice Guideline for CFD simulation of flows in the urban environment: A summary,” Int. J. Environ. Pollut. 44(1-4), 419 (2011).

${ }^{41}$ S. B. Pope, Turbulent Flows (Cambridge University Press, 2000).

${ }^{42}$ I. B. Celik, Z. N. Cehreli, and I. Yavuz, "Index of resolution quality for large eddy simulations," J. Fluid Eng. 127(5), 949 (2005).

${ }^{43}$ J. K. R. D. Moser and N. N. Mansour, "Direct numerical simulation of turbulent channel flow up to $\operatorname{Re}_{\tau}=590$," Phys. Fluids 11(4), 943 (1999).

${ }^{44}$ Y. Jongwoo, J. Y. Yoo, and H. Choi, "Direct numerical simulation of heated vertical air flows in fully developed turbulent mixed convection," Int. J. Heat Mass Transfer 46(9), 1613 (2003).

${ }^{45}$ D. E. Newland, Random Vibrations, Spectral and Wavelet Analysis (John Wiley \& Sons Inc., 1993).

${ }^{46}$ D. C. Montgomery and G. C. Runger, Applied Statistics and Probability for Engineers (John Wiley \& Sons, 2010).

${ }^{47}$ J. Lighthill, Waves in Fluids (Cambridge University Press, 2001). 